\title{
Neue Inschriften aus dem phrygischen Hochland
}

\author{
N. Eda AKYÜREK ŞAHİN* - Hüseyin UZUNOĞLU**
}

Yazılıkaya - in der Literatur durch W. M. Ramsay bekannt als Midasstadt ${ }^{1}$ - wurde Anfang der 1800er Jahre von dem englischen Offizier W. M. Leake entdeckt. Im 19. und 20. Jh. war diese Gegend ein beliebtes Ziel für zahlreiche westliche Forschungsreisende; C. Texier, J. R. Steuart, A. Mordtmann, G. Perrot, W. M. Ramsay, G. Radet, A. Körte und E. Brandenburg können als deren Vorreiter genannt werden. Gemäß dem vorherrschenden Zeitgeist waren diese ersten Forscher nicht nur darum bemüht, die prächtigen Felsgräber des phrygischen Hochlandes, allen voran das Midas-Monument, sondern auch alle phrygischen und griechischen Inschriften, die sie fanden, zu dokumentieren"2. W. M. Ramsay konnte die Veröffentlichung des für seine "The cities and bishoprics of Phrygia"-Reihe geplanten Bandes "Highland Phrygia" nicht verwirklichen, da es ihm nicht möglich war, sich länger in dieser Gegend Phrygiens aufzuhalten, obwohl er derjenige war, der unter den genannten Forschern die meisten epigraphischen Funde gemacht hatte. Dadurch blieb diese Gegend aus Sicht der Epigraphik weitgehend unberührt, bis C. H. Emilie Haspels nach dem Ende des zweiten Weltkrieges mit Ihren Forschungen begann und ihre Ergebnisse 1971 in zwei Bänden veröffentlichte. Ihr Werk enthielt 154 Inschriften aus phrygischer, griechischer und byzantinischer Zeit, von denen lediglich 30 bereits zuvor bekannt waren. Die restlichen Inschriften wurden von ihr neu entdeckt. Da Haspels' Ziel gemäß ihrer eigenen Aussage die Erforschung der phrygischen Epoche dieser Gegend war ${ }^{3}$, legte sie einen einfachen Katalog der Inschriften ohne Kommentar vor. Im Zuge ihrer Bewertung der hellenistischen, römischen und christlichen Epochen in Abschnitt III bis V des Buches bezog sich Haspels des Öfteren auf die Inschriften.

* Prof. Dr. N. Eda Akyürek Şahin, Universität Akdeniz, Fakultät für Human- und Sozialwissenschaften, Fach für Antike Sprachen und Kulturen, TR-07058 Kampus, Antalya (edasahin@akdeniz.edu.tr; https://orcid.org/0000-0002-5993-0566).

** Asst. Prof. Dr. Hüseyin Uzunoğlu, Universität Akdeniz, Fakultät für Human- und Sozialwissenschaften, Fach für Antike Sprachen und Kulturen, TR-07058 Kampus, Antalya (huseyinuzunoglu@akdeniz.edu. tr; https://orcid.org/0000-0001-7707-4647).

Unser herzlicher Dank gilt Doç. Dr. Rahşan Tamsü Polat und Dr. Öğretim Üyesi Yusuf Polat für die Erteilung ihrer Erlaubnis, an den epigraphischen Funden aus ihrer Oberflächenforschung "Eskișehir-Frig Vadisi” zu arbeiten. Wir bedanken uns ferner bei Herrn Gregor Staab sehr herzlich, der das Manuskript gelesen hat und inhaltlich wie sprachlich mit wertvollen Hinweisen verbesserte.

${ }^{1}$ Der alte Name des Gebiets, in dem sich Yazılıkaya befindet, wird in einem kürzlich erschienenen Artikel von Susanne Berndt als Leonton Kephalai vorgeschlagen, der zuvor mit Afyon identifiziert wurde, s. Berndt 2018.

${ }^{2}$ Texier 1839, 154-156; Steuart 1842, 12; Perrot 1872, 137, Nr. 94; Ramsay 1887, 498-499 = IGR IV, 592; Anderson 1897, 422-423, Nr. 21-22; Körte 1898, 154; Mommsen 1897, 660-663 = ILS 8845a/b; Ramsay 1908, 397, Nr. 28; Ramsay 1918, 135-136; MAMA I, Nr. 387-390; MAMA V, S. 174-175, Nr. Z.B. 82-86; MAMA VI, 390-399.

${ }^{3}$ Haspels 1971, viii: "My aim has been the publication of the Phrygian remains of the Highlands. But I have also included the later, post-Phrygian material, which is noticeable wherever one goes". 
Haspels Werk füllte die Lücke in der Literatur, die Ramsay hinterließ, was ihre Arbeit besonders verdienstvoll machte.

Auch nach Haspels wurden in der Gegend Forschungen in unterschiedlichen Umfang fortgesetzt. Davon ist insbesondere das zwischen 2001 und 2012 von Taciser T. Sivas und Hakan Sivas durchgeführte Projekte zu nennen ${ }^{4}$. Das phrygische Hochland ist, anders als erwartet, reich auch an epigraphischen Relikten, von denen an dieser Stelle fünf neue und eine bekannte (Nr. 5) griechische Inschriften vorgelegt werden. ${ }^{5}$

Bei zweien der Inschriften (Nr. 1 und 2) handelt es sich um Weihinschriften, die an den Gott Megistos Hosios geweiht wurden. Es kann angenommen werden, dass diese Gottheit mit Hosios und Dikaios in Beziehung steht, die in Phrygien weit verbreitet sind. Zwei weitere Inschriften (Nr. 3 und 4) sind Epigramme und die letzten beiden (Nr. 5 und 6) einfache Grabinschriften.

Die Inschriften Nr. 1, 2, 5 sowie 6 wurden im Dorf Başara im Landkreis Han der Provinz Eskişehir, Nr. 3 in Yazılıkaya und Nr. 4 im Dorf Ağlarca im Landkreis Han gefunden ${ }^{6}$. Betrachtet man den Charakter der Buchstaben in den vorliegenden Inschriften, können diese generell in die römische Kaiserzeit (2.-3. Jhr. n. Chr.) datiert werden.

${ }^{4} \mathrm{~S}$. für die detaillierte archäologische Forschungsgeschichte des phrygischen Hochlandes Tüfekçi Sivas 1999, 11-24; Berndt 2002; Tüfekçi Sivas 2007; Tüfekçi Sivas - Sivas 2007; Haspels 2009; Polat 2009; Tüfekçi Sivas 2012, 112-159; Tamsü Polat 2018; s. für manche Inschriften, die aus dem phrygischen Hochland Yazıdere stammen und nach Haspels veröffentlicht wurden SEG 37, 1168 (Madrid Archäologisches Museum); Akyürek Şahin 2007, 95-97, Nr. 75 (momentan im Archäologischen Museum Afyon befindlich); s. für Nakoleia und Umgebung Drew-Bear 1978, 32-52; Akyürek Şahin 2006a, 107-110, Nr. 28-33. s. für den Corpus, in dem sich die Inschriften des $7 \mathrm{~km}$ nordöstlich von Nakoleia liegendem Heiligtum bei dem Dorf Yazıdere, Akyürek Şahin 2006b.

${ }^{5}$ Wir haben die Inschriften selbst nicht gesehen, aber die ausgezeichneten Fotos ausgewertet.

${ }^{6}$ Es ist schwierig zu bestimmen, zum Territorium welcher Stadt diese Siedlungen gehörten. Im phrygischen Hochland gibt es zwei antike Städte mit Polisstatus: Meiros/Meros (Dorf Malatça/Döğer) und Metropolis (Kümbet). Es ist bekannt, dass Meiros in der Kaiserzeit eine Katoikia war (Haspels 1971, 165) und in Bezug auf die Lage nicht in Frage kommt. Wenn es stimmt, dass Metropolis seit der hellenistischen Zeit eine Polis war, wie L. Robert annimt, besteht kein Zweifel daran, dass es der am besten geeignete Kandidat ist, s. Robert 1980, 298-299. Vgl. Waelkens 1986, 120-121; Belke - Mersich 1990, 340-341 (zu Meiros s. auch İznik 2006, 145-147). Yazllıkaya und die umliegenden Siedlungen werden in den SGO unter Metropolis geführt. Die Wahrscheinlichkeit, dass sich das Gebiet von Nakoleia bis hierher ausdehnt, ist gering. Es wird jedoch angenommen, dass das Dorf Başara zumindest in der Römerzeit zu Nakoleia gehörte, s. Alp 2010, 22. Eine spätantike Grabinschrift aus dem Dorf Başara zeigt, dass es sich bei dieser Siedlung um das $\chi \omega$ píov

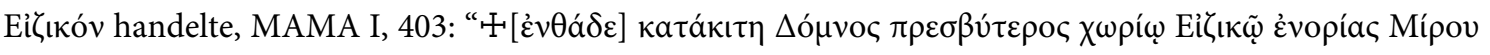

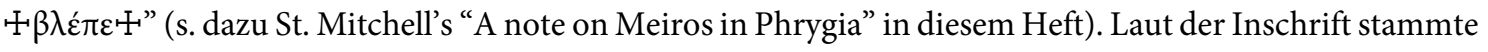
der Verstorbene aus Meiros. Allerdings wird dies von den Autoren der Monumenta Asiae Minoris Antiqua zurecht nicht als Beweis dafür, dass sich die Grenzen von Meiros bis hierher erstreckten, gewertet. 


\section{Weihung an den Gott Megistos Hosios}

Altar aus Kalkstein; FO: Dorf Başara im Landkreis Han bei Eskişehir; H: 74 cm; Br: 28 cm; T: 27 $\mathrm{cm}$; Bh: $2,5 \mathrm{~cm}$.

Vgl. Akyürek Şahin - Uzunoğlu 2020, 217, Nr. A.9.

Der Altar ist oben und unten sowie an allen Ecken und Rändern angebrochen. Die Oberfläche ist stark beschädigt. Der Sockel fehlt. Die ersten zwei Zeilen der Inschrift befinden sich auf dem hohen Aufsatz. Die restlichen drei Zeilen folgen auf dem Schaft. Unterhalb der Inschrift befindet sich das Relief einer Heliosbüste, die auf einem kugelförmigen Sockel mit einer Basis dargestellt ist. Allerdings sind die Büste und das Gesicht der Gottheit stark verwittert. Sorgfältig gearbeitete Strahlen umringen das Haupt. Von der Inschrift fehlen die Buchstaben an den Bruchkanten.

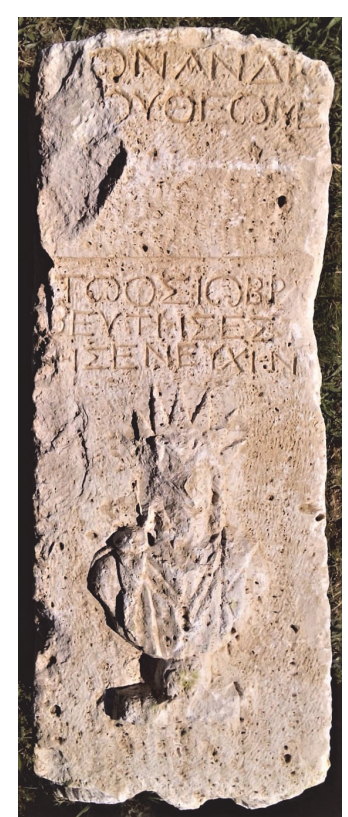

Abb. $1 a$

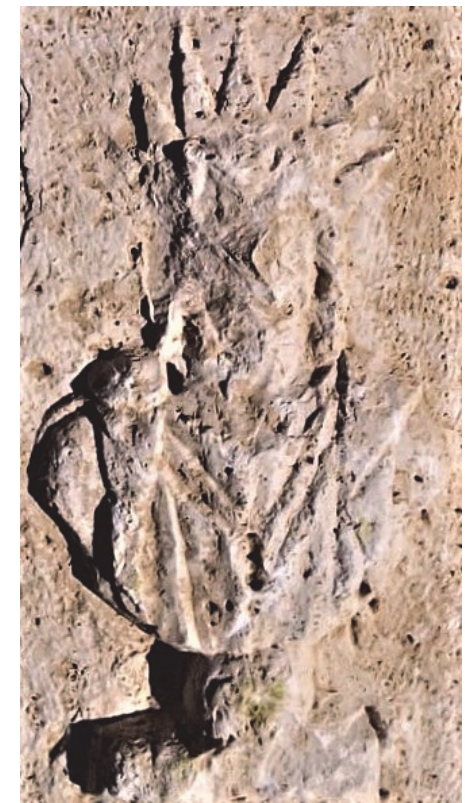

$A b b .1 b$

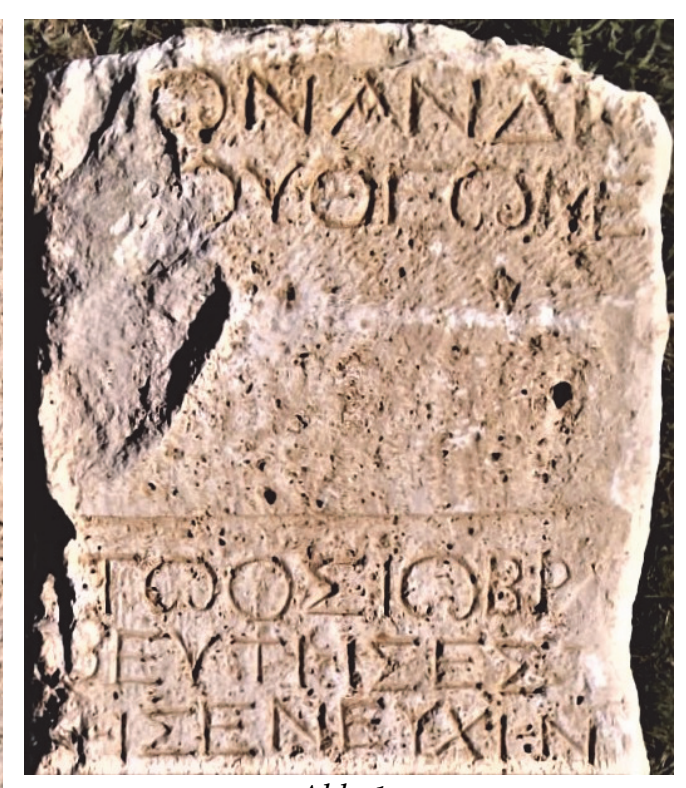

Abb. $1 c$

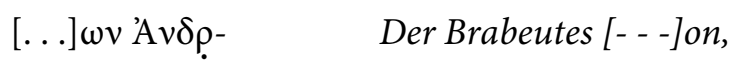

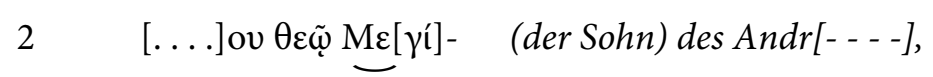

$$
\begin{aligned}
& \text { Profil hat es dem Gott }
\end{aligned}
$$

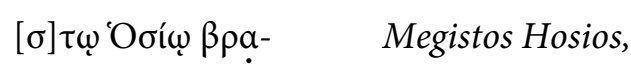

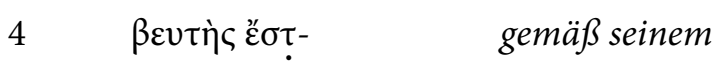

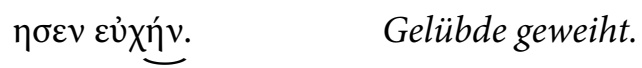

Zeile 1: [. . .] $\omega v$. Hier fehlen zwei oder drei Buchstaben. An dieser Stelle dürfte wohl ein Name, ähnlich dem in Midaion belegten $\Delta \mathrm{t} \delta \omega v$ (CIG, Nr. 4124), dem in Orkistos (Çifteler-Ortaköy) belegten $\Xi \varepsilon ́ v \omega v$ (Waelkens 1986, Nr. 586 und LGPN VC, 323) oder dem in Ankyra belegten Mí $\delta \omega v$ (MAMA X, Nr. 449), gestanden haben. Es ist aber möglich, dass hier ein deutlich längerer Name stand, der in einer Zeile darüber beginnt.

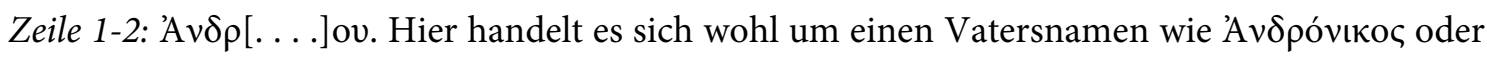

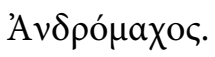




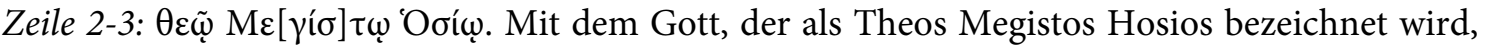
dürfte wohl Hosios kai Dikaios gemeint sein. Diese gehören zu den wichtigsten Gottheiten in Phrygien, die u.a. im Territorium Dorylaion verehrt wurden. Die Namen von Hosios kai Dikaios erscheinen in den Inschriften in vielen verschiedenen und komplexen Formen, s. Ricl 1992a, 7377 und Ricl 2008, 563-564. Das Wesen des Kultes ist von Ricl ausführlich behandelt worden (Ricl 1992a, 93-95 und Ricl 2008, 567). Ricl's Studien haben gezeigt, dass die Inschriften und Denkmäler von Hosios und Dikaios in Lydien im Allgemeinen auf einen einzigen Gott ( $\theta \varepsilon o ́ c)$ hinweisen. Es versteht sich jedoch, dass die Situation in Phrygien anders ist und Hosios kai Dikaios öfters als zwei getrennte Götter (auch ikonographisch, vgl. z.B. Akyürek Şahin - Uzunoğlu 2020, 213214, Nr. 18) wahrgenommen werden. Die in Phrygien gefundenen Inschriften geben jedoch keine sehr klaren Informationen über das Wesen dieses Kultes. Hosios kai Dikaios taucht hier zum ersten Mal in dieser Form, also als Megistos und ohne den Zusatz von Dikaios auf. ${ }^{7}$ In dieser und der nächsten Inschrift (Nr. 2) erscheint die Gottheit nur als Hosios (ohne Dikaios). Die alleinige Bezeichnung "Hosios" ist im gesamten "Hosios-kai-Dikaios-Katalog" bei 16 Inschriften bekannt. ${ }^{8}$

Es ist auch interessant, dass ein Braubeutes und ein Priester (s. unten Nr. 2) jeweils einen Altar für diesen Gott weihten. Die Altäre sind einander sehr ähnlich. Sie standen wohl im selben Heiligtum im Dorf Başara oder in seiner Umgebung. Vielleicht wurden beide Weihungen im selben Kontext aufgestellt, was wir aber nicht weiter klären können.

Ein im Dorf Başara gefundener Altar, dessen Inschrift zerbrochen und verloren ist, zeigt eine Figur auf seiner Vorderseite, die ein Zepter in Händen hält und deren Kopf mit Strahlen umgeben ist. Obwohl diese Figur von W. M. Calder, dem Herausgeber, als Hosios kai Dikaios identifiziert wurde (vgl. ebenda Nr. 398), ähnelt sie eher Helios.

Auf der Vorderseite des Altars sticht eine besonders ordentlich gearbeitete Heliosbüste hervor. Es ist mittlerweile bekannt, dass der Gott Helios eine wichtige Stellung im "Hosios-kai-Dikaios-Kult" innehatte. Heliosbüsten ähnlich dieser hier begegnen sehr häufig auf Weihsteinen, auf deren Inschriften manchmal auch der Name des Gottes geschrieben steht. ${ }^{9}$

Durch umfängliche Forschungen und Veröffentlichungen ${ }^{10}$ in den letzten Jahren hat sich unser Wissen um den Hosios kai Dikaios Kult deutlich vergrößert. In erster Linie hat sich nun deutlich

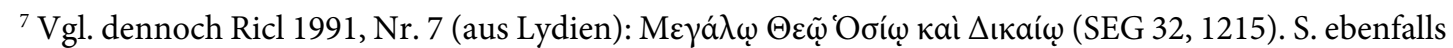

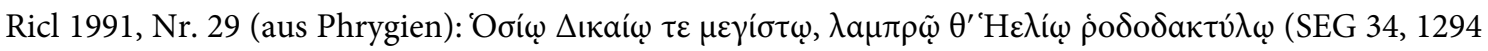

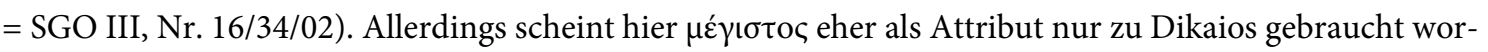
den zu sein.

${ }^{8}$ Vgl. Ricl 1992a, 74 und Ricl 2008, 564.

${ }^{9}$ Für eine detaillierte neue Veröffentlichung bzgl. der Platzes und der Darstellung des Gottes Helios in der Ikonographie des Hosios kai Dikaios Kultes s. Erten 2018, 204-214.

${ }^{10}$ Für grundlegende Informationen zum Kult s. Ricl 1990; Ricl 1991; Ricl 1992a; Ricl 1992b; Lochman 2003, 198-207; Ricl 2008. Nach Ricl 2008 wurden ebenfalls neue Weihungen bzw. mit dieser Thematik zusammenhängende Arbeiten veröffentlicht, s. Akyürek Şahin - Uzunoğlu 2020, 189-230 und Coşkun 2020, 111-133. Für eine neue Liste der nach Ricl 2008 veröffentlichten Inschriften s. Akyürek Şahin - Uzunoğlu 2020, 216-220: Addenda. Die zwei hier veröffentlichten Weihungen befinden sich ebenfalls in dieser Liste. 
gezeigt, dass die Gottheit nicht überall in Anatolien, sondern vor allem in Phrygien und im südlichen Mysia Abbaitis (gebräuchlicher Maionia ${ }^{11}=$ Nordostlydien) intensiv verehrt wurde. Es wurden auch vereinzelte Funde außerhalb dieser Regionen und außerhalb Kleinasiens gefunden. Die bis heute entdeckten knapp 200 Inschriften, zu denen auch die hier veröffentlichten zählen, lassen keinen Zweifel daran, dass der Kult seinen Ursprung in Phrygien hatte. Die komplexe Religionsvielfalt zeigt sich auch im Phrygien der Kaiserzeit wie in vielen anderen Regionen bereits allein in der Betrachtung dieses einen Kultes. Hosios kai Dikaios wird oft gemeinsam mit vielen anderen Gottheiten angebetet oder dargestellt.

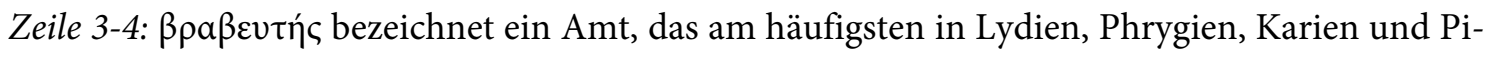
sidien in Erscheinung trat und oft in den Siedlungen im Landesinneren (selten in den Stadtzentren) bekleidet wurde. Im Etymologicum Magnum steht geschrieben, dass diese Personen die Aufgabe hatten, einen triumphierenden Agon mit einem Siegeskranz oder einem Preis zu ehren,

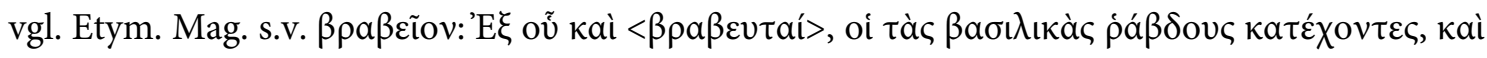

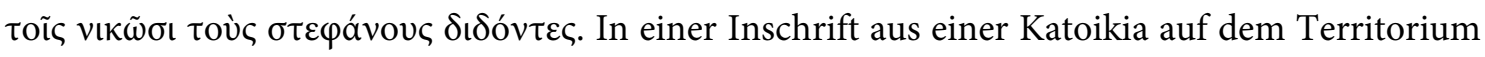
von Hierakaisareia ist zu lesen, dass die Brabeutai ihr Amt für ein Jahr bekleiden und für die Herstellung eines Ehrenkranzes für einen einheimischen Wohltäter bzw. eines Priesters des Kai-

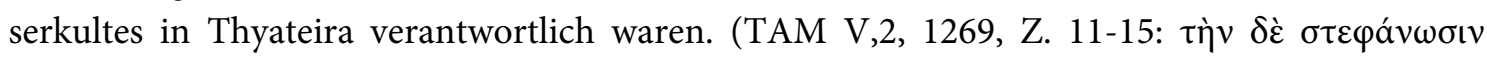

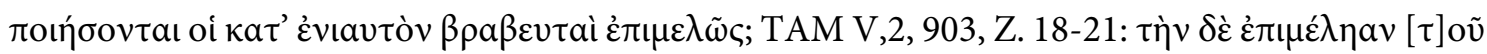

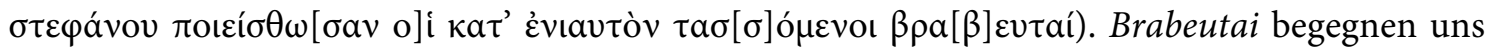
gleichzeitig auch als ein eponymes Amt, s. für ein Beispiel aus Dorylaion Frei 1988, 15, Nr. 5N =

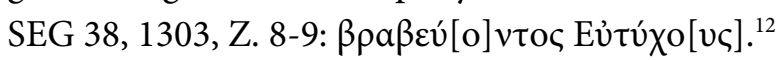

Wir hätten in der Inschrift die Bezeichnung als brabeutes direkt hinter dem Namen des Vaters des Weihenden erwartet. Allerdings steht die Bezeichnung hinter dem Namen des Gottes, also etwas entfernt von dem Namen des Weihenden. Eine derartige Schreibweise, nämlich dass zwei zueinander gehörige Worte nicht direkt nebeneinanderstehen, begegnet aber auch in anderen Inschriften.

\section{Weihung an dem Gott Megistos Hosios}

Altar aus Kalkstein; FO: Dorf Başara bei der Kreisstadt Han bei Eskişehir; H: 83 cm; Br: 25 cm; H: $33 \mathrm{~cm}$; Bh: $2-4 \mathrm{~cm}$.

Vgl. Akyürek Şahin - Uzunoğlu 2020, 217-218, Nr. A.10.

Der Altar ist an allen Ecken und Seiten angebrochen und an der Oberfläche stark beschädigt. Der Großteil des Aufsatzes und des Sockels fehlt. Auf der Vorderseite sind auf dem Schaft die Spuren einer stark verwitterten Büste erkennbar. Oberhalb der Büste wurden die ersten drei Zeilen der Inschrift geschrieben. Die Inschrift, die sich seitlich am Altar in zwei Zeilen fortsetzt, wurde von

\footnotetext{
${ }^{11}$ Vgl. Naour 1981; Lochman 2003, 30 Anm. 31; 204 Anm. 27-28.

${ }^{12}$ Für detailliertere Informationen zu den Brabeutes vgl. Keil - Premerstein 1908, 55-56; Magie 1950, 1026 Anm. 70; Herrmann 1962, 9-10; Frei 1988, 15-16 Anm. 18-19; Mitchell 1993, 185; Schuler 1998, 238-

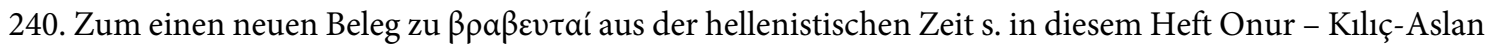
2021, 20-22.
} 
beiden Seiten beschädigt. Unter den zwei verwitterten Zeilen ist ein ebenfalls stark verwittertes Kranzrelief erkennbar.

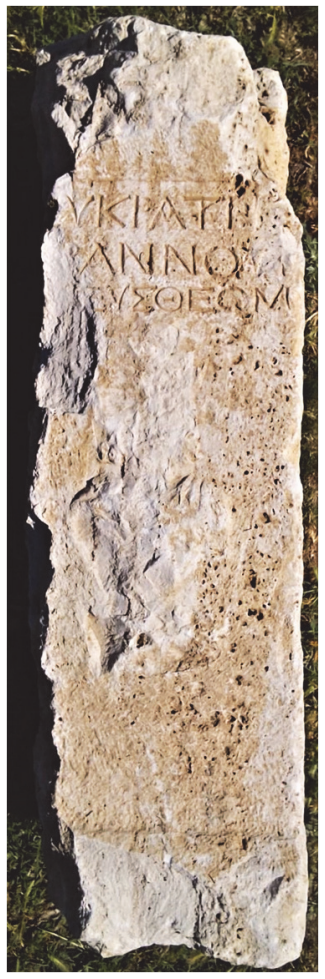

Abb. 2a: Vorderseite

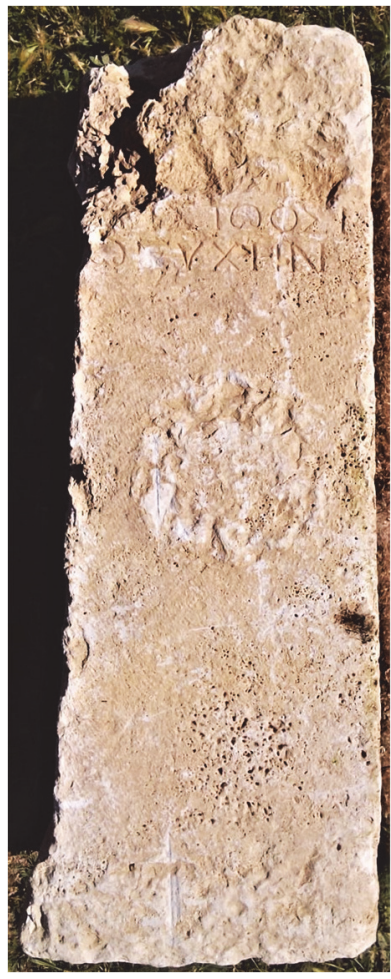

Abb. 2b: rechte Nebenseite

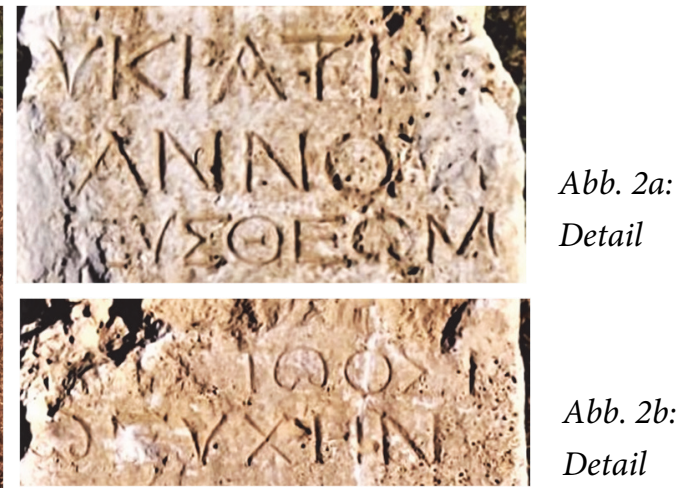

Die Inschrift auf der Vorderseite

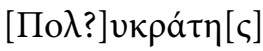

2 [Tu]pávvou í-

$[\varepsilon \rho] \varepsilon \dot{c} \theta \varepsilon \varepsilon \tilde{\omega} \mathrm{M}-$

Die Inschrift auf der rechten Nebenseite

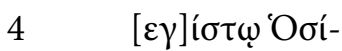

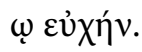

Der Priester Polykrates?, der Sohn des Tyrannos, hat diesen Altar dem Gott Megistos Hosios geweiht.

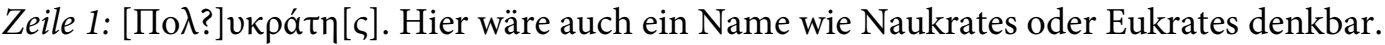

Zeile 2: Zu einem anderen Tyrannos, den möglicherweise Leiter eines Bakchischen Vereins, welcher dem Hosios (und) Dikaios in Erfüllung eines Gelübdes ein Altar aufgestellt hat, s. Güney 2018, Nr. 1 = Coşkun 2020, 120 .

Zeile 2-3: í[ $\varepsilon \rho] \varepsilon u ́ c$. Es ist naheliegend, dass es ein "Megistos-Hosios-Heiligtum" in der Nähe des Fundortes gab, da in der Inschrift der Name eines Priesters dieses Kultes genannt wurde.

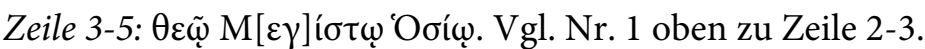

\section{Dankepigramm des Pasikrates an Apollon}

Großer Altar aus Kalkstein; FO: Yazılıkaya. Er steht dort im Garten des sogenanten „Turist evi“; H: ca. 145 cm; Br: 40-50 cm; T: 40 cm; Bh: Auf dem Aufsatz: 8 cm, Auf dem Schaft: 3-4 cm. 


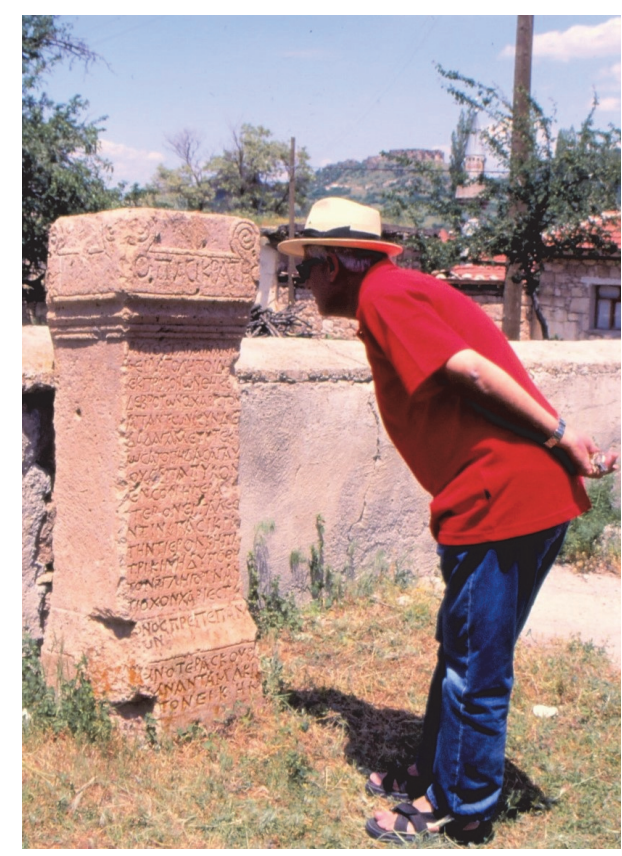

Abb. 3a: Der verstorbene Prof. Dr. Sencer Şahin beim Betrachten dieser Inschrift, 1998.
Der Altar besitzt einen langen Schaft. Es sind zahlreiche kleine Risse, Sprünge und Löcher in den Ecken, den Rändern und der Oberfläche zu sehen. Der große und hohe Aufsatz, der durch mehrere Profile mit dem Schaft verbunden ist, hat Akrotere mit Voluten in den oberen Ecken. Der Schaft ist durch ein breites Profil mit dem Sockel, der im linken Eck abgebrochen ist, verbunden. Die Vorderseite des Steines ist gänzlich mit einer Inschrift ausgefüllt. Diese beginnt auf dem Aufsatz mit einer einzelnen Zeile, die in größerer Schrift den Namen des Stifters präsentiert. Auf dem Sockel sind drei Zeilen zu lesen. Der Steinmetz hat Hilfslinien für seine Arbeit benutzt.

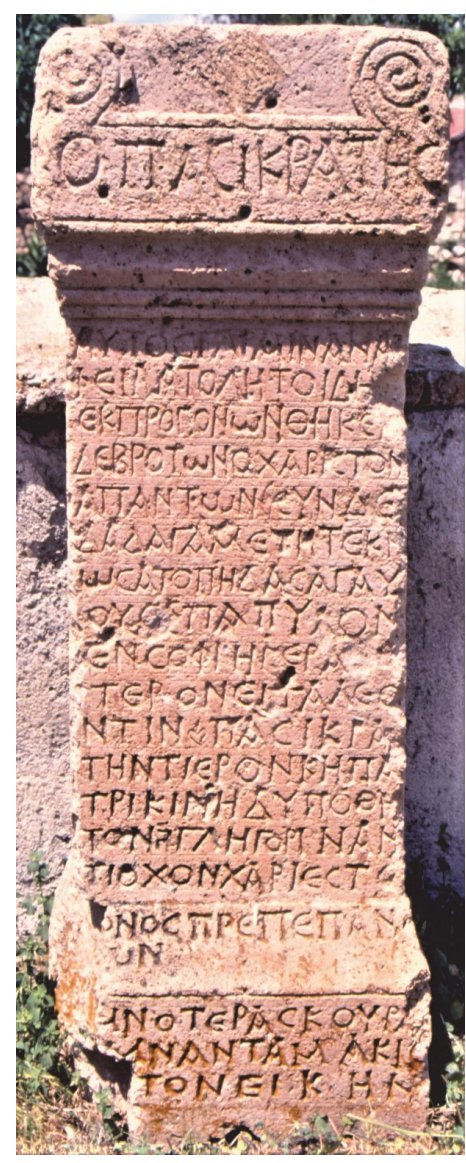

$A b b .3 b$

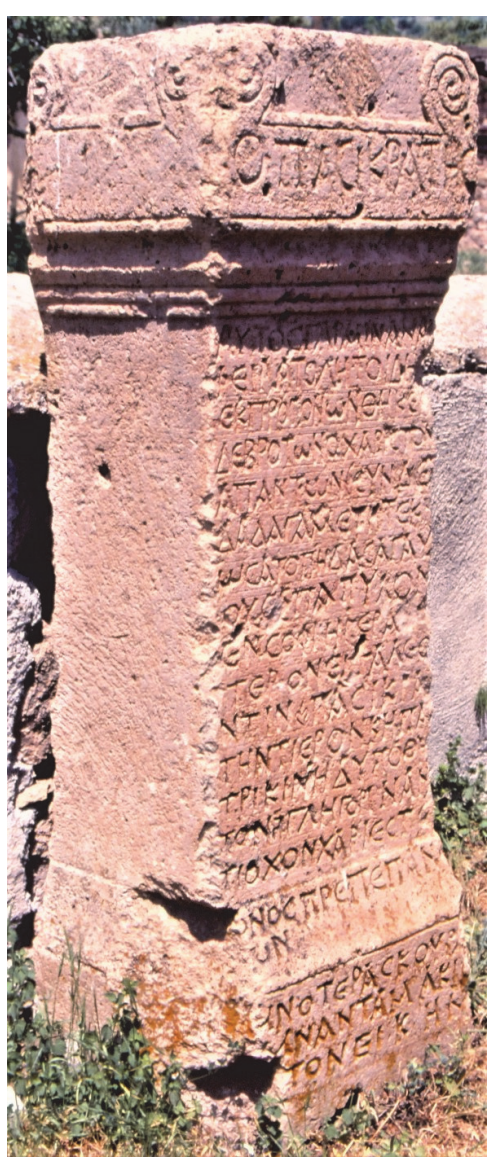

$A b b .3 c$

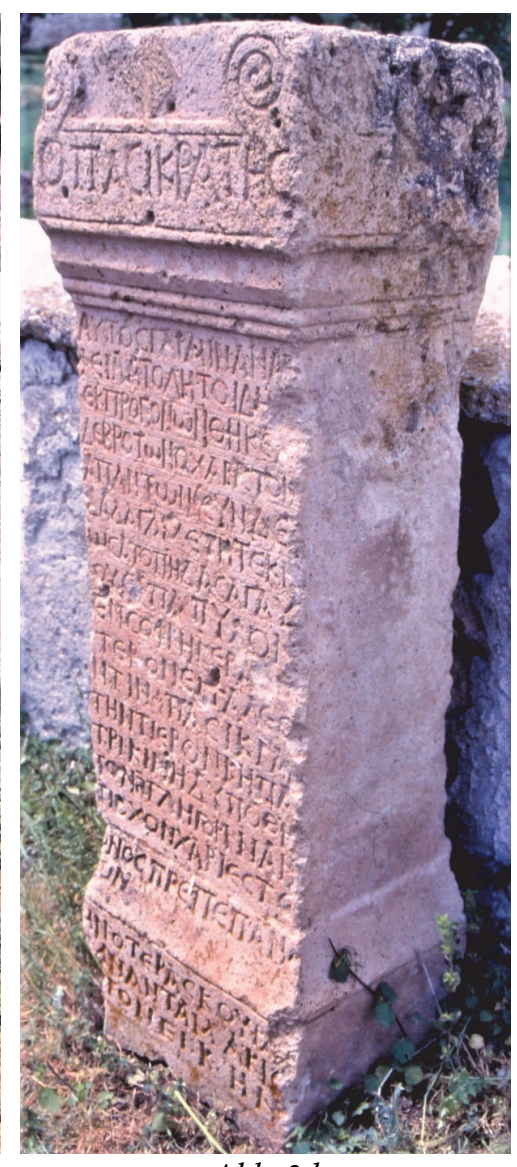

Abb. $3 d$ 


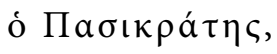

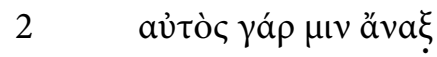

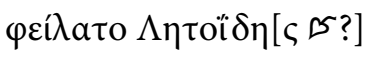

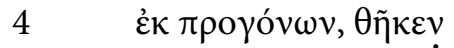

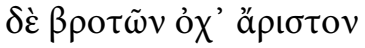

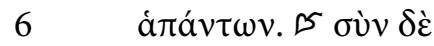

$\Delta \alpha \delta a \underline{\gamma} \gamma \alpha \mu \varepsilon \tau \underline{n} \tilde{\tau} \tau \kappa \nu-$

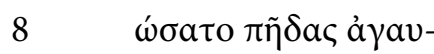

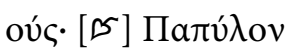

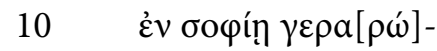

$\tau \varepsilon \rho o v, \varepsilon i ̃ \tau \alpha \Lambda \varepsilon_{0}-$

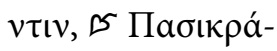

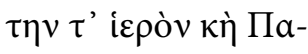

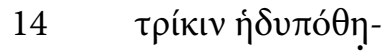

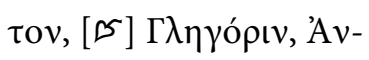

16

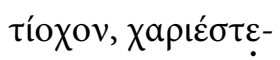

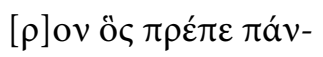

18

$[\tau] \omega v, \quad v a c$

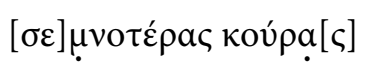

20

[ $\Delta$ ó] $\mu v \alpha v \tau^{\prime}$ ă $\mu \alpha \kappa<\grave{\eta}>\sum$ -

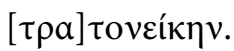
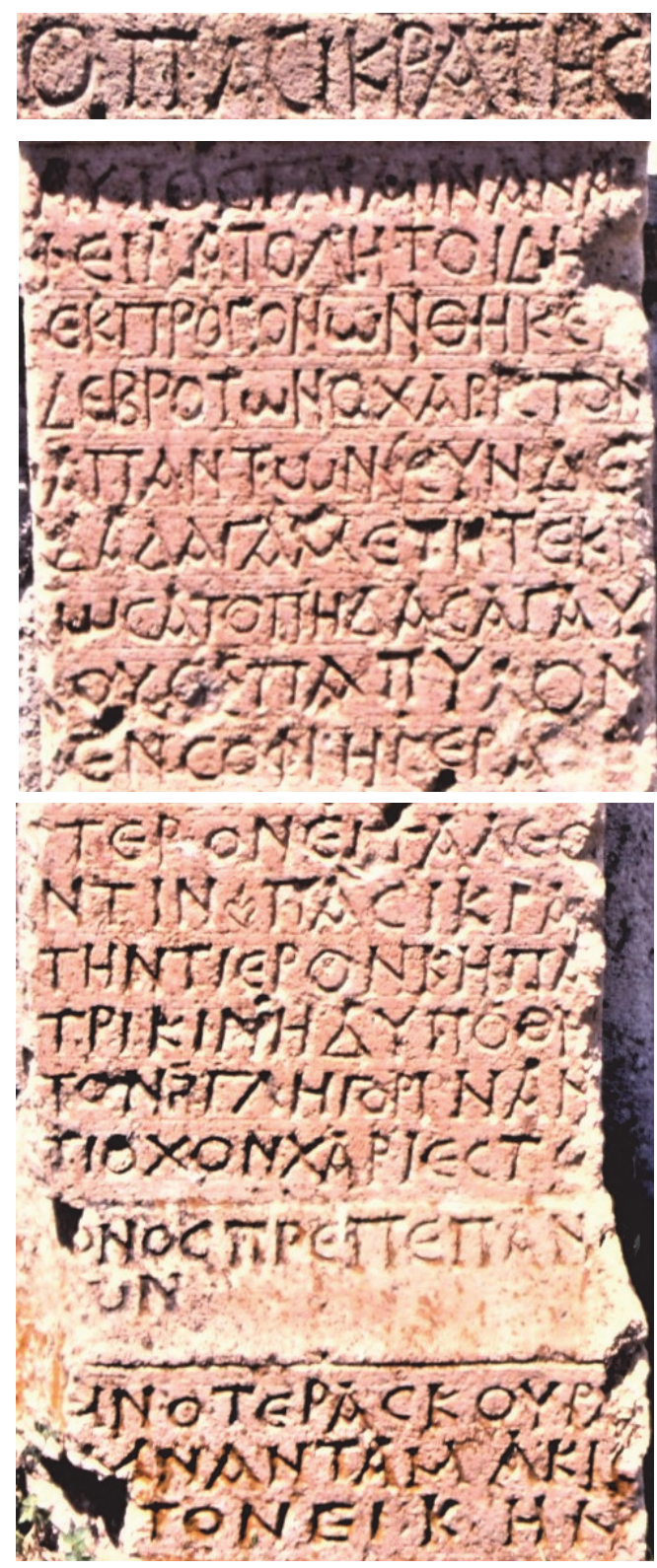

Abb. $3 e$

Pasikrates (hat dies geweiht). Denn der Herr, der Sohn der Leto selbst, erkor ihn sich von den Vorfahren her zum Liebling (und) machte ihn zum bei weitem Besten unter allen Sterblichen. Zusammen mit seiner Frau Dada zeugte er prächtige Kinder: Den Papylos, der an Weisheit noch älter ist, dann den Leontios, den Gott geweihten Pasikrates und den schmerzlich vermissten Patrikios, Gregorios (und) den recht anmutigen Antiochos, der an Glanz alle übertrifft, die ehrbaren Mädchen, Domna zusammen mit Stratonike.

Das Gedicht besteht aus 1 Pentameter (G. Staab) (Vers 1) und 6 Hexametern. Das erste Wort der Inschrift gehört nicht zum Versmaß.
Vers 1

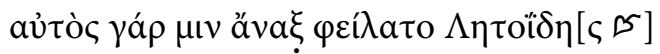
Vers 2

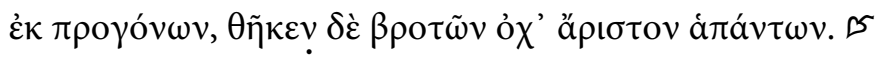
Vers 3

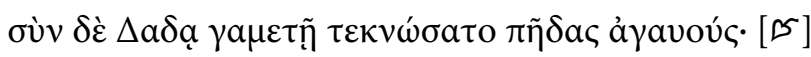
Vers 4

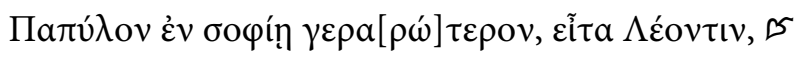




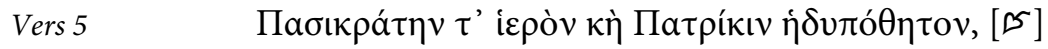

$$
\begin{aligned}
& \text { Vers } 6 \quad \Gamma \lambda \eta \gamma \text { ó }
\end{aligned}
$$

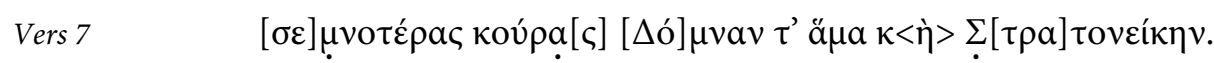

Zeile 2: Die Lesung von åvał stammt von G. Staab. Das Adjektiv ävał wird seit Homer sowohl für Apollon als auch für viele Götter oder Göttinnen verwendet, s. Hemberg 1959, 7-11.

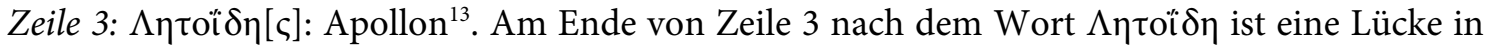
der Größe von ca. zwei Buchstaben. Wahrscheinlich standen hier ein Sigma und danach ein Efeublatt. Wenn man das gesamte Gedicht betrachtet, sieht man, dass an verschiedenen Stellen (Z. 6, $9,12,15$ ? u. evtl. 3) einzelne Efeublätter ( $(5)$ abgebildet wurden. Bei näherer Betrachtung erkennt man, dass diese Blätter genutzt wurden, um die Versenden zu kennzeichnen. In der 15. Zeile wurde dieses Zeichen nicht in Form eines Blattes, sondern eher eines Stigmas genutzt, wobei dennoch deutlich erkennbar ist, dass eigentlich ein Blatt gemeint ist. Dieses Zeichen befindet sich am Ende der Zeile 18 nicht, da es hier sowieso eine große Lücke (vacat) gibt. Auch am Ende von Vers 7 (Zeile 21) gibt es kein Blatt, da das Gedicht nach diesem Vers endet.

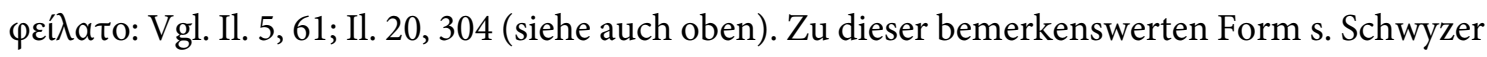

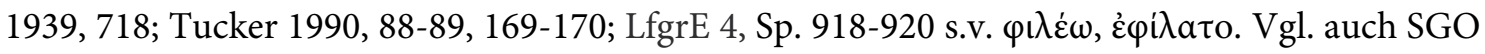

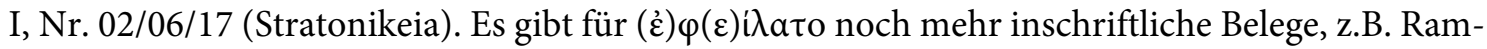
say 1897, 606 Nr. 495, Z. 20, (Sebaste in Phrygien; IGR 4, 682): غ̇ழ ments), aber auch darüber hinaus, z.B. im dorischen Sprachraum: à $\varphi$ ì $\alpha \tau$.

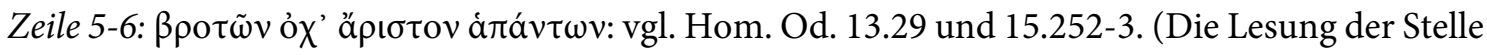

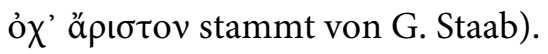

Zeile 6: Versenden werden durch Efeublätter oder vacat (Z. 18) markiert; vgl. z.B. Akyürek Şahin - Uzunoğlu 2018, Nr. 1.

Zeile 7: $\Delta a \delta \alpha$ ist ein häufiger Frauenname und gehört zur Gruppe der "Lallnamen" vgl. Zgusta 1964, § 244-1 und LGPN VC, 104.

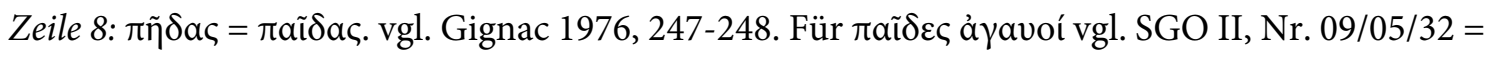
SEG 51, 1713.

Zeile 9: Ab dieser Zeile werden die Kinder des Paares, Pasikrates und Dada, einzeln aufgezählt.

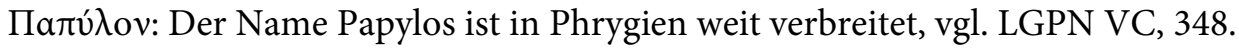

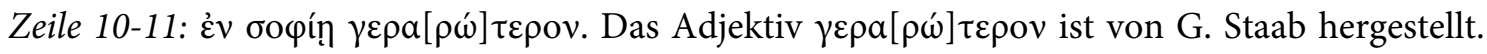
Ein ähnlicher Ausdruck ist in einem christlichen Grabepigramm aus Ankyra belegt, vgl. SGO III,

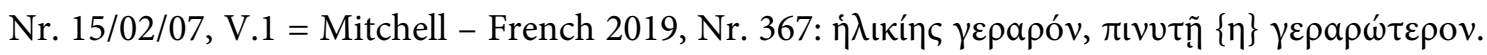
Vgl. zum einen vergleichbaren Ausdruck von "jung, aber reifer in der Weisheit zu sein” (ebenfalls

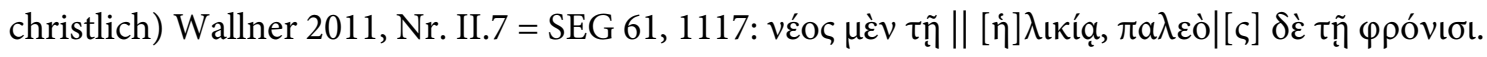

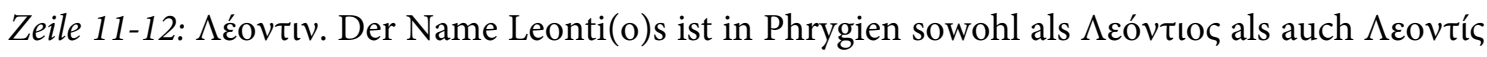
belegt, vgl. LGPN VC, 244. Der Ausfall von -o- ist schon seit dem 2. Jh. eine sehr häufige Erscheinung.

\footnotetext{
${ }^{13}$ Letoides wird auch als Personenname gebraucht, s. Le Bas - Waddington 1972, Nr. 837 (Dorf Hac1 in der Nähe von Aizanoi/Phrygien), vgl. LGPN VC, 246.
} 


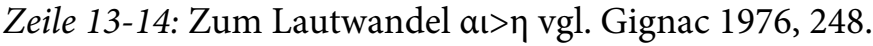

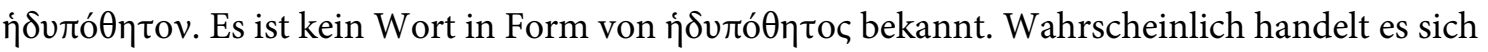

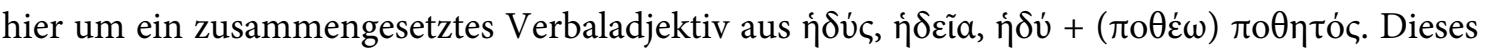
Adjektiv scheint das erste Mal in dieser Inschrift belegt zu sein. G. Staab vermutet, dass Patrikios schon verstorben war.

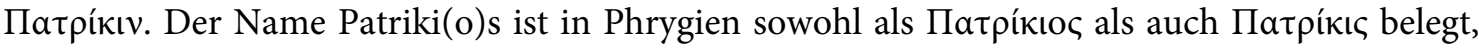
vgl. LGPN VC, 351.

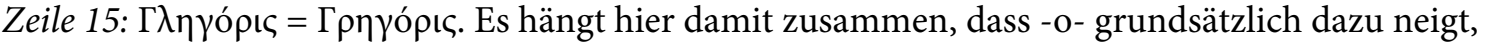
nach -t- auszufallen (wie Patrikis, Leontis, Apollinaris, usw.). Der Mann heißt im Nominativ

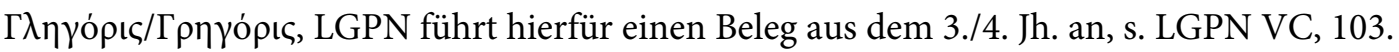

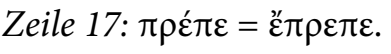

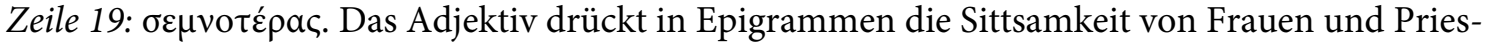
terinnen aus, s. Pircher 1979, 36-37 (G. Staab).

\section{Grabepigramm für Karikos}

Große Tür-Stele aus Marmor; FO: Dorf Ağlarca bei der Kreisstadt Han bei Eskişehir; H: 120 cm; Br: $137 \mathrm{~cm}$; T: $40 \mathrm{~cm}$; Bh: 2,5-3 cm.

Der aus einem großen Block geschnittene Stein ist in Form einer Stele gearbeitet, äußerst tief und wird heute als Brunnenstein verwendet. Die Stele hat zwar keinen dreieckigen Giebel, aber einen Aufsatz mit mehreren Profilen. Auf dem Schaft direkt unter dem Aufsatz ist eine fünfzeilige, die Oberfläche bis zu den Rändern ausfüllende, sorgfältig geschriebene Inschrift zu lesen. Abgesehen von kleinen Beschädigungen ist sie vollständig erhalten. Unterhalb der Inschrift sind zwei nebeneinanderliegende Figurentafeln in Türform tief eingearbeitet worden (vgl. Waelkens 1986, 9: Typ K. Langrechteckige Türsteine). Die Türen sind in vier gleich große Kassetten getrennt. In jede dieser Türen sind verschiedene Reliefmotive dargestellt. Während im oberen rechten Abschnitt der linken Tür ein zierlicher Krug in Form einer Oinochoe dargestellt ist, befindet sich im linken Abschnitt die Darstellung einer Flasche mit langem Hals in Form eines Unguentarium. Links im unteren Abschnitt befindet sich wahrscheinlich ebenfalls ein Gefäß in Form eines Kruges, rechts ist ein Wollkorbmotiv zu erkennen. Diese Motive weisen auf ein Frauengrab hin. Im linken Abschnitt der rechten Tür sieht man das Motiv einer bauchigen langen Flasche, im rechten Abschnitt eine senkrechte Raute. Auf der unteren linken Tafel befindet sich ein Winzermesser, rechts auf der unteren Tafel die Darstellung eines Eselkopfes. Die Abbildungen auf dieser Tür deuten auf einen Mann hin. Die Grabstele wurde für ein Ehepaar entworfen. Allerdings ist aus der Inschrift erkennbar, dass es sich bei dem Verstorbenen um einen Mann namens Karikos handelt. An den Türrahmen sind Pflanzenornamente zu erkennen. Auf der Oberseite der Stele wurden drei Dübellöcher eingelassen, die höchstwahrscheinlich zur Befestigung der in der Inschrift erwähnten Löwenstatuen dienten, vgl. hier Abb. 4e. 

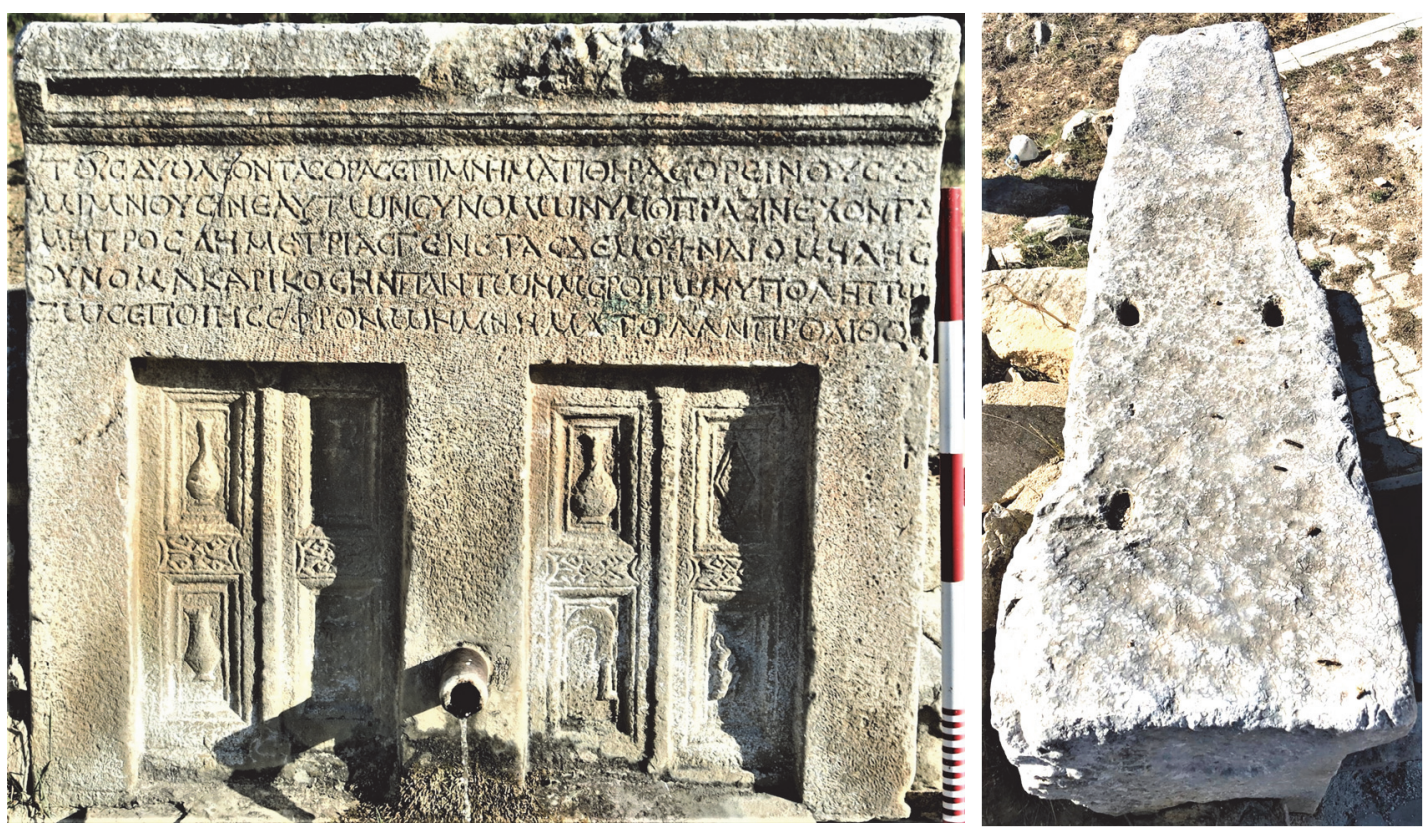

$A b b .4 a$

Abb. 4b: Ansicht von oben

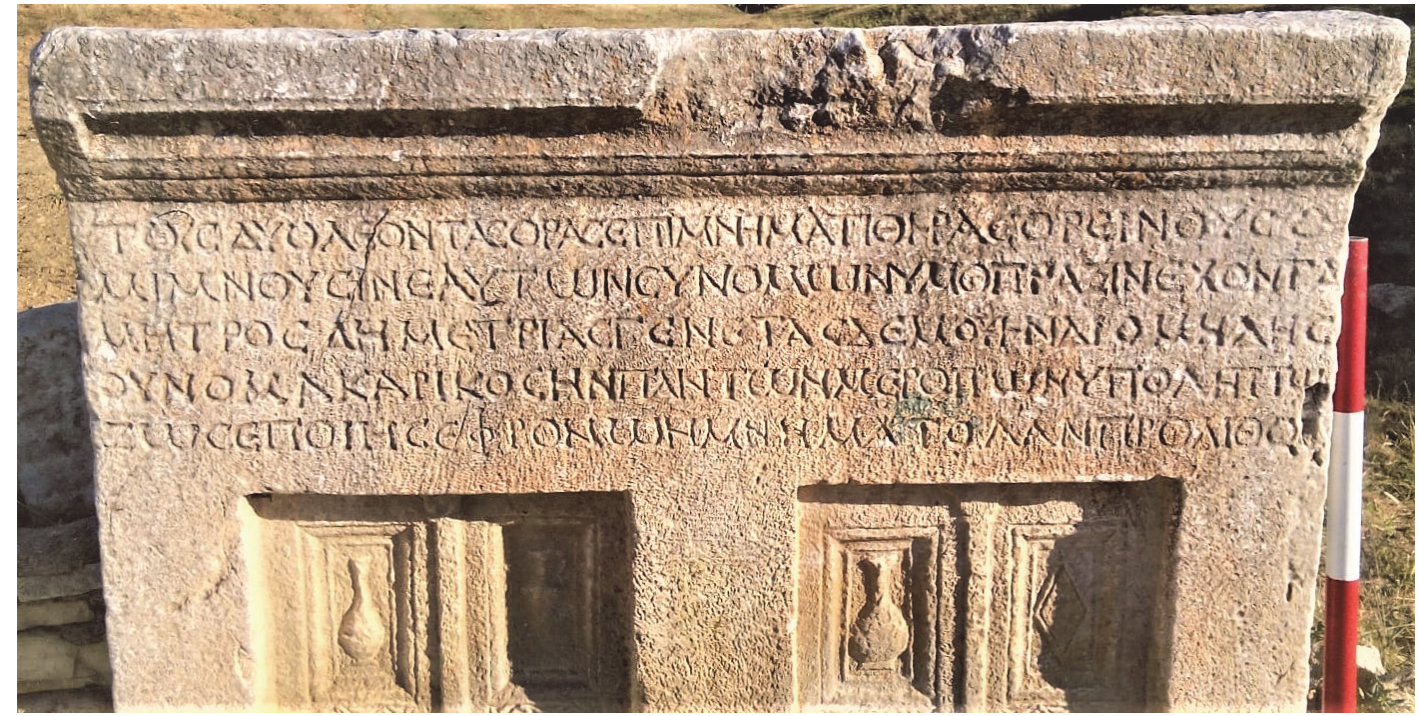

$A b b .4 c$

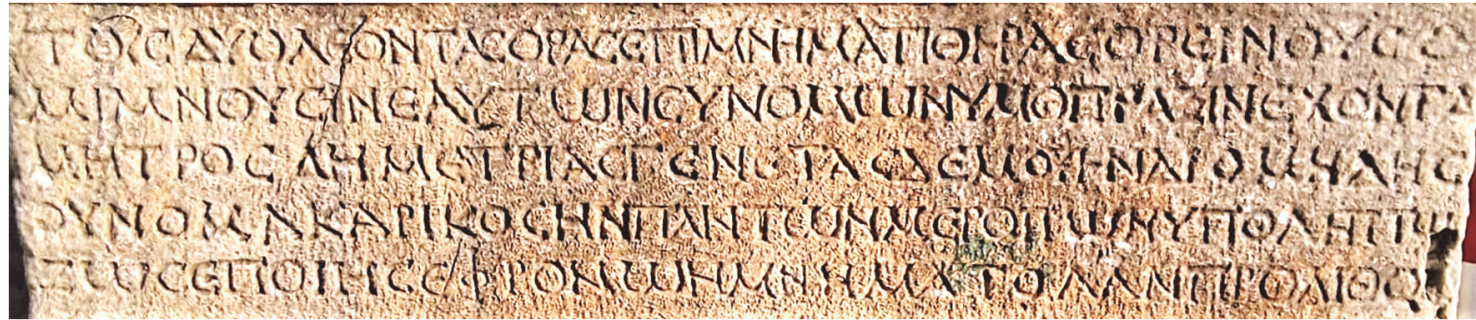

Abb. $4 d$

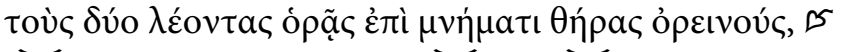

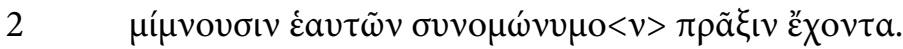

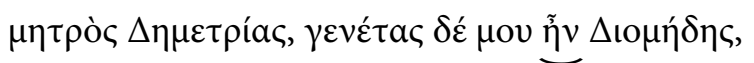

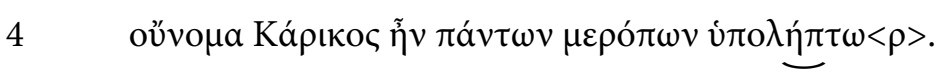

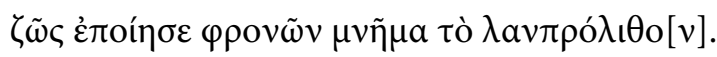


Du siehst die zwei Löwen auf diesem Denkmal, Tiere, die in den Bergen leben; sie warten auf jemanden, der etwas tut, das mit ihrem Namen identisch ist. (Ich bin geboren) von Demetria, meiner Mutter, und mein Erzeuger war Diomedes. Mein Name war Karikos, der Helfer aller Sterblichen. $\mathrm{Zu}$ Lebzeiten hat er (= Karikos) dieses hervorstechende Steindenkmal in vollem Bewusstsein errichten lassen.

Das Gedicht besteht aus drei Hexametern und dann einem Distichon.

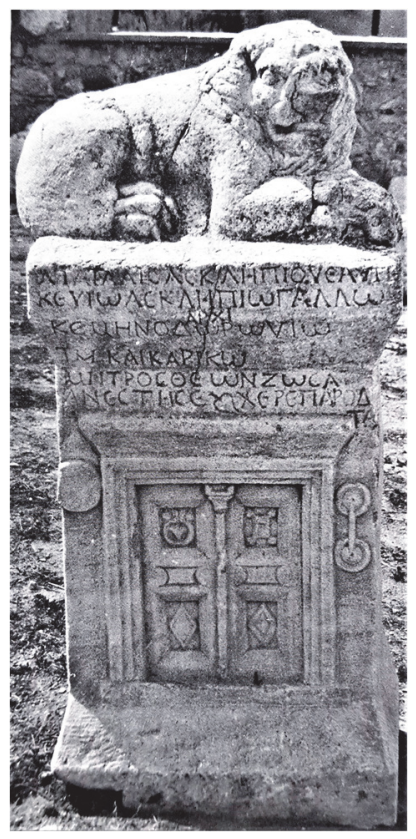

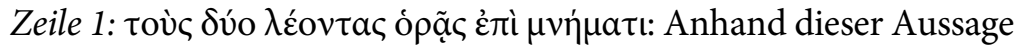
ist eindeutig erkennbar, dass sich auf dem Grab zwei Löwenstatuen befanden. Diese sind heute nicht mehr vorhanden und wurden separat auf das Grabmal montiert, wie man an den Dübellöchern, die zur Befestigung genutzt wurden, erkennen kann, vgl. Abb. 4b. Es sind weitere Beispiele zu Löwen-Statuen auf Grabmälern bekannt, s. z. B. Pfuhl - Möbius 1979, Nr. 526, Nr. 2152 sowie Nr. 2168, Tafel 310; Waelkens 1986, Nr. 753 Tafel 96; I.Pessinous, Nr. 64 (= einer von den Grabinhabern dieses Denkmals hiess auch Karikos; hier Abb. 4e); s. auch MAMA I, Nr. 401 (wie das hier neu vorgelegte Monument aus dem Dorf Başara). Löwenstatuen auf Grabmälern dienten als Abschreckung gegen Grabräuber und hatten zudem eine apotropäische (Unheil abwehrende) Funktion, s. Kubińska 1968, 61; Lochman 2003, 210 Anm. 5.

Abb. 4e: Waelkens 1986, Nr. 753 Tafel 96 (aus Pessinus).

Ein weiteres Beispiel stammt aus der Siedlung Syrna an der Rhodischen Peraia. Aus einem Gedicht auf dem Linteau eines Grabmales in Pyramidenform geht hervor, dass die Löwen das Grab eines gewissen Diagoras, der im Krieg verstorben war, bewachen, SGO I, Nr. 01/06/01. Der Umstand, dass in der Nähe dieses Grabmales eine beschädigte Löwenstatue gefunden wurde, bewahrheitet das Gedicht auch aus archäologischer Sicht. Ein besonders prächtiges Beispiel zur Thematik ist ein kürzlich in Kotiaeion gefundener, aus dem Jahre 171/172 n. Chr. stammender Grabaltar

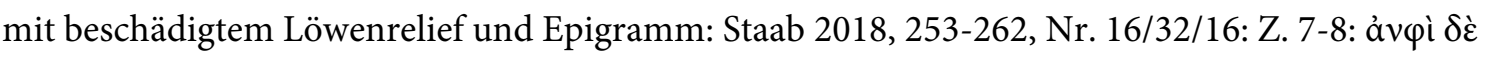

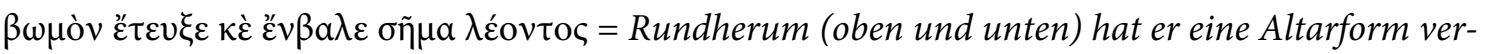
fertigt und darauf das Bild eines Löwen eingesetzt.

Der Zusammenhang zwischen Grabbauten und Löwendarstellungen zeigt sich deutlich in einem Epigramm des berühmten Dichters Antipatros aus Sidon, der zwischen dem Ende des 2. Jhd. und

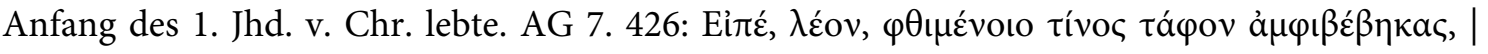

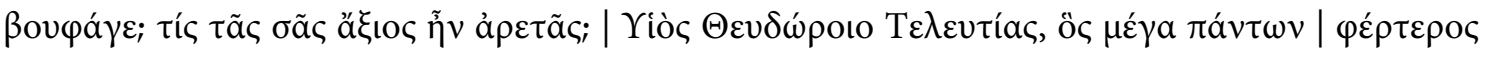

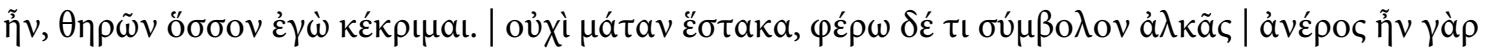

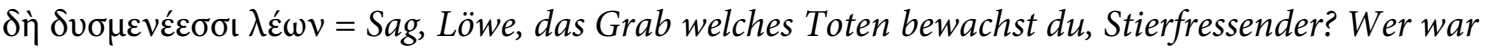
deiner Tüchtigkeit würdig? - Der Sohn des Theodoros Teleutias, der bei Weitem mächtiger als alle war, wie ich mächtiger als die (anderen) Tiere bin. Nicht vergeblich stehe ich hier, ich zeige die Wehrhaftigkeit des Mannes an; denn er war den Feinden wahrhaftig ein Löwe (Übersetzung: Christian 2015, 22). 
Ein weiteres Gedicht wurde von einem Dichter aus Kanytellis (Kanlıdivane) in Kilikien, inspiriert durch die Werke des Antipatros, verfasst, s. SGO IV, Nr. 19/10/01; Garulli 2012, 142-150; Christian 2015, 21-22. Allerdings dienten die hier auf einer runden Säule dargestellte Löwenstatute einem anderen Zweck: Sie sollte das Land eines gewissen Sandaios bewachen und erfüllte so die Aufgabe eines Grenzsteines.

Es gibt noch weitere Epigramme, in denen Grabmäler und Löwen miteinander verbunden werden: SGO I, Nr. 01/20/40 (Miletos); SEG 58, 1553 = Staab 2018, 170-173, Nr. 02/16/01 (Museum von Denizli); für eine detaillierte Übersicht zu dieser Thematik, s. Staab 2018, 121-124; 258; des Weiteren s. Robert 1937, 394-398.

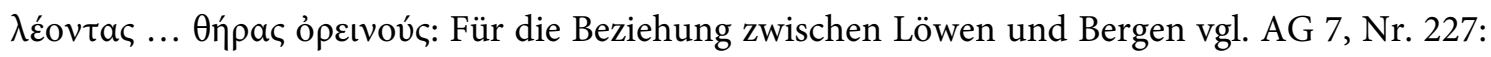

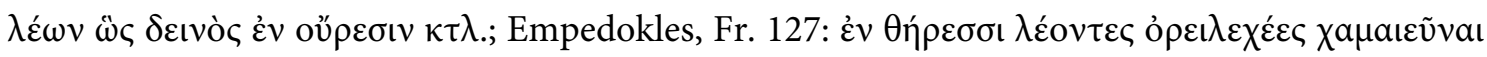

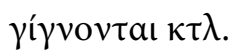

Zeile 2: $\mu 1 \mu v \omega$ ist eine Form des Verbes $\mu \varepsilon \dot{v} \omega$, die in Poesie und Epos vorkommt.

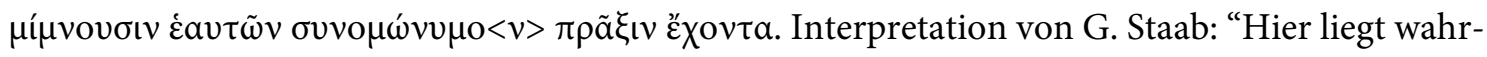

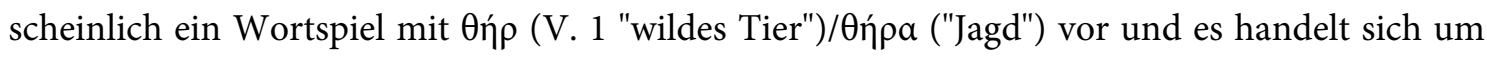
eine Bildbeschreibung der verschollenen Steinskulptur, wenn gesagt wird, daß die Löwen auf

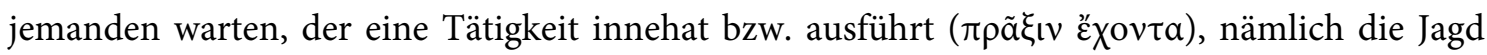
( $\theta \eta ́ \rho \alpha)$, die deren Benennung als "wildes Tier" ( $\theta \eta ́ \rho)$ gleich ist. Mit dem Abwarten kann auch darauf angespielt sein, daß die dargestellten Löwen auf dem zu Lebzeiten errichteten Grabmal auf Karikos warten, bis er als Verstorbener bei ihnen weilen wird."

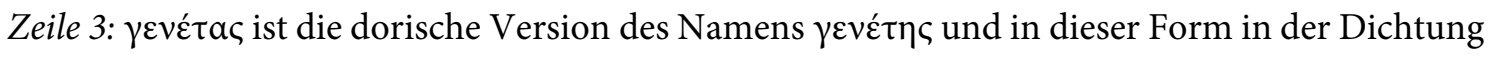
weit verbreitet.

Zeile 4: $\dot{\pi} \pi \mathrm{\lambda} \eta \dot{\pi} \tau \omega<\rho>$ (Lesung und Hinweise von G. Staab): Das Wort kommt zweimal vor: s. SGO II, Nr. 10/02/11 und 10/02/28 (beide aus Kaisareia/Hadrianupolis). Auf dem Stein nach Omega gibt es keinen Platz für das Rho. Vielleicht hatte der Steinmetz das Rho unterhalb des Omegas geschrieben, wo es dort einen kleinen Bruch gibt.

Das zusammengesetzte Adjektiv $\lambda a v \pi \rho o ́ \lambda \_\theta o c$ ist zwar bislang nicht belegt, aber in seiner Bedeutung eindeutig.

\section{Grabstein des Solon und der Nana}

Große Tür-Stele aus Tufstein; FO: Dorf Başara bei Han bei Eskişehir; H: ca. 94 cm; Br: 50 cm; T: $37 \mathrm{~cm}$; Bh: $4 \mathrm{~cm}$.

Literatur: MAMA I, 209 Nr. 400 (ohne Foto aber mit einer groben Zeichnung der Stele).

Die zwei großen Löcher auf dem Stein, die erst im Nachhinein eingelassen wurden, deuten darauf hin, dass der Stein in moderner Zeit als Brunnen genutzt wurde. Der aus einem großen Block herausgeschnittene Stein wurde in Form einer Stele gearbeitet und ist nicht sehr tief. Der Sockel des Grabmales ist abgebrochen und nicht mehr vorhanden. Die untere linke Ecke ist ebenfalls abgebrochen. Da die Oberfläche des Steines stark abgenutzt ist, sind die Inschrift und die Figuren nur mit Mühe zu erkennen. Ein dreieckiger Giebel ist nicht vorhanden. Das Grabmal hat einen dicken und mit einem Profil verbundenen Aufsatz. Auf dem Profil befindet sich eine einzeilige 
Inschrift, deren unterer Teil fehlt. Auf der Oberfläche der Stele befinden sich zwei viereckige nebeneinanderliegende Figurentafeln, die nicht besonders tief eingelassen wurden. Die oberen Ränder des Aufsatzes sind in Form eines dreieckigen Giebels gearbeitet. Auf den Giebeln befinden sich äußerst auffällige und große Akrotere. Die Türen beider Tafeln sind in vier gleich große Kassetten unterteilt, in denen sich jeweils unterschiedliche Reliefmotive befinden. Die Türen sind unten abgebrochen und die Ornamente fehlen. Im oberen linken Bereich der linken Tür befindet sich ein Türknauf. Der rechte Bereich ist stark vom Brunnenloch beschädigt worden. In den oberen rechten Teil der rechten Tür wurde ein Schlüssellochmotiv eingearbeitet. Der linke Teil dieser Seite wurde ebenfalls durch ein Brunnenloch beschädigt. Auch dieses Grabmal wurde für ein Ehepaar entworfen. Vgl. Waelkens 1986, 9: Typ K. Langrechteckige Türsteine.

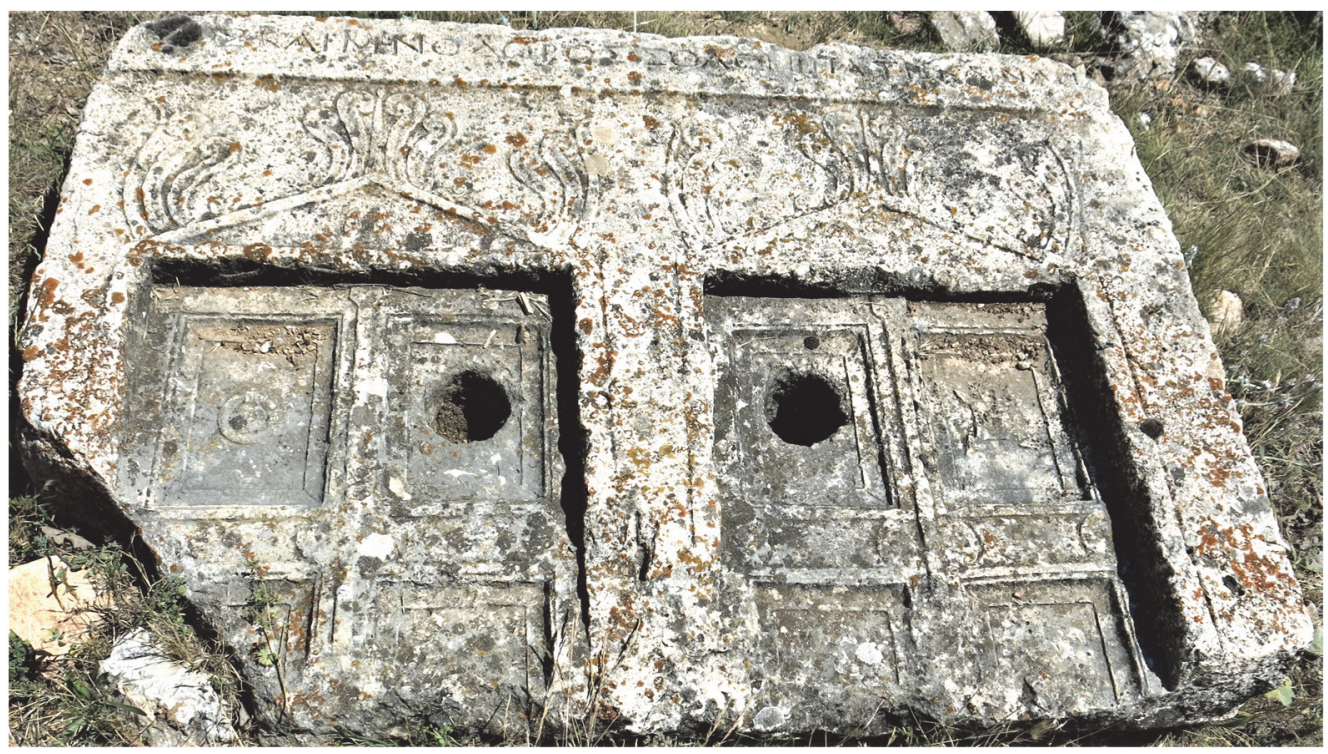

$A b b .5 a$

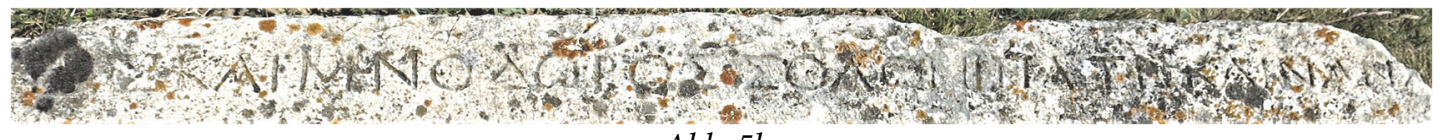
$A b b .5 b$

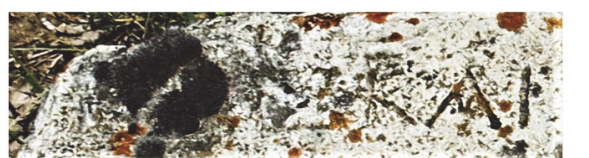

$A b b 5 c$

$A b b .5 d$

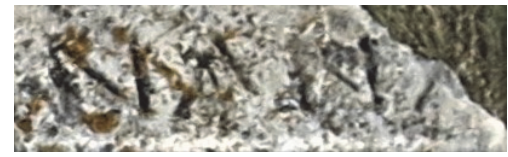

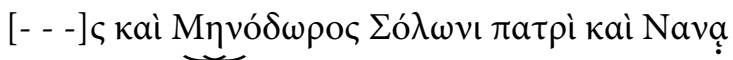

- - -s und Menodoros für ihren Vater Solon und (ihre Mutter) Nana.

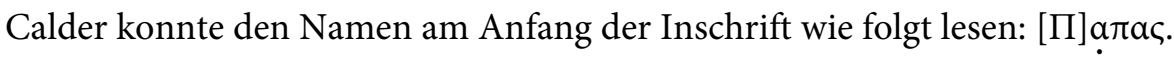

Nava. Für den Lallnamen Nana vgl. Zgusta 1964, \$1013-1 und LGPN VC, 308.

Der untere Teil der Inschrift fehlt. Die Inschrift wird wohl wie folgt weitergegangen sein: $[\mu \eta \tau \rho \grave{~}$

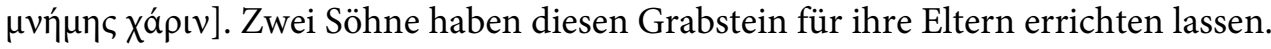

\section{Grabstein}

Große Tür-Stele aus Marmor; FO: Dorf Başara bei Han bei Eskişehir; H: 143 cm; Br: 115 cm; T: $56 \mathrm{~cm} ; \mathrm{Bh}: 2,5 \mathrm{~cm}$. 
Von der Grabstele ist lediglich ein großes Fragment übriggeblieben. Dieses ist von fast allen Seiten angebrochen. Auf dem unteren Bereich der Oberfläche des Steines befinden sich zwei viereckige Nischen, in die Figuren eingearbeitet wurden. Die linke der nicht sehr tiefen Nischen ist komplett abgebrochen und fehlt. In der rechten Nische lassen sich zwei nebeneinanderstehende stark verwitterte Figuren, eine männliche und eine weibliche, erkennen.
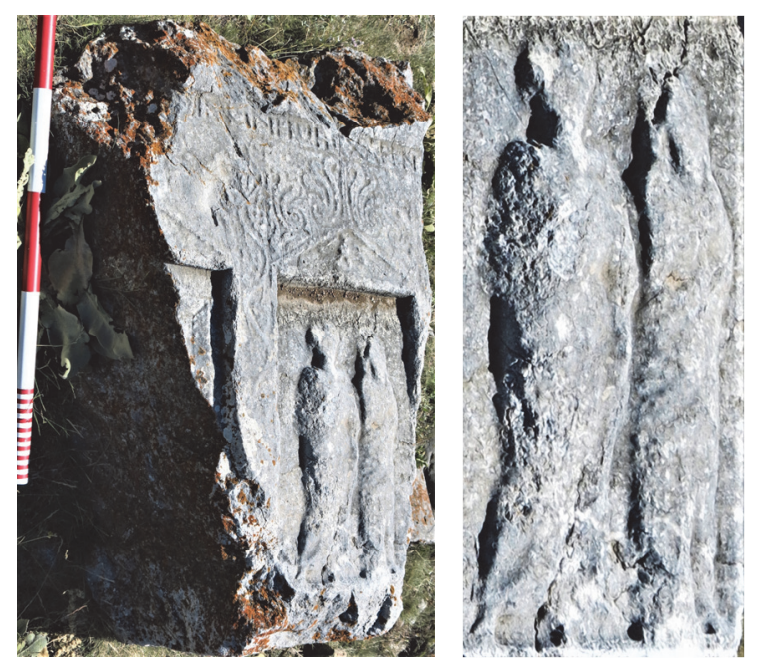

Die Details der Figuren sind nicht mehr vorhanden. Über den Nischen befinden sich, eher fein ausgearbeitet, Giebel, die fast einen aufgemalten Eindruck hinterlassen. Die Akrotere sind sehr prunkvoll geschmückt. Oberhalb der Giebel befindet sich ein Fragment der letzten Inschriftenzeile. Der Großteil der Inschrift fehlt. Vgl. Waelkens 1986, 9: Typ K. Langrechteckige Türsteine.

Abb. $6 c$

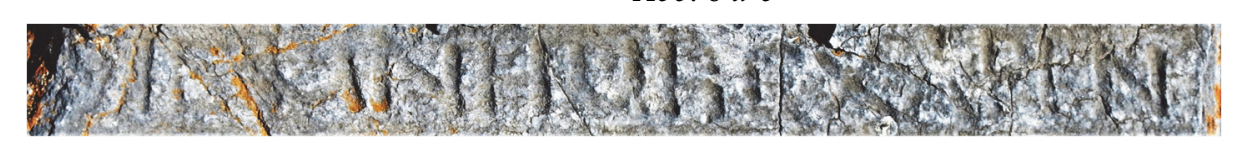

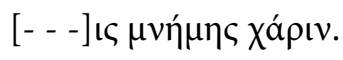
des Andenkens wegen - - -

\section{Namensverzeichnis}

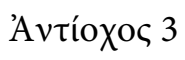

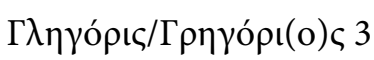

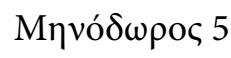

Nava 5

$\Delta \alpha \delta a 3$

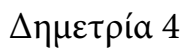

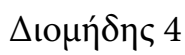

$\Delta$ ó $\mu v a 3$

Káрıкос 4

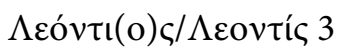

\section{Bibliographie}

AG

Akyürek Şahin 2006a
Патас 5

Палúdos 3

Пабเкра́тๆৎ 3

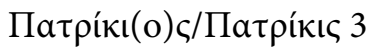

$\Sigma o ́ \lambda \omega v 5$

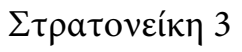

Túpavvoc 2

Fragmente

A $v \delta \rho[\ldots]$ os 1

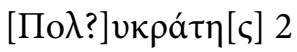

$[\ldots] \omega \vee 1$

\section{Gottheiten}

"Oбıৎ 1, 2

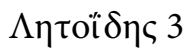

Anthologia Graecae. Greek Anthology, Vol. II with an English translation by W. R. Paton, London 1919.

N. E. Akyürek Şahin, Phrygia'dan Yeni Zeus Bronton Adakları, Arkeoloji ve Sanat 122, 2006, 89-124. 
Akyürek Şahin 2006b

Akyürek Şahin 2007

Akyürek Şahin -

Uzunoğlu 2018

Akyürek Şahin -

Uzunoğlu 2020

Alp 2010

Anderson 1897

Belke - Mersich 1990

Berndt 2002

Berndt 2018

Christian 2015

Coşkun 2020

Drew-Bear 1978

Empedokles

Erten 2018

Etym. Mag.

Frei 1988

Garulli 2012

Gignac 1976

Güney 2018
N. E. Akyürek Şahin, Yazıdere (Seyitgazi) Kutsal Alanı ve Adak Yazıtları, İstanbul 2006.

N. E. Akyürek Şahin, Neue Votivsteine aus dem Museum von Afyon, Gephyra 4, 2007, 59-115.

N. E. Akyürek Şahin - H. Uzunoğlu, Eskişehir Müzesi'nden Yeni Yazitlar, in: M. Arslan - F. Baz (eds.), Arkeoloji, Tarih ve Epigrafi'nin Arasında: Prof. Dr. Vedat Çelgin'in 68. Doğum Günü Onuruna Makaleler, İstanbul 2018, 33-67.

N. E. Akyürek Şahin - H. Uzunoğlu, Neue Weihungen an Hosios kai Dikaos aus dem Museum von Eskişehir, Gephyra 19, 2020, 189-230.

A. O. Alp, Eskişehir, Başara Köyü Kazılarında Bulunan Bizans Dönemi Kiliseleri, in: K. Pektaş et al., XIII. Ortaçağ ve Türk Dönemi Kazıları ve Sanat Tarihi Araştırmaları Sempozyumu Bildirileri, 14-16 Ekim 2009, İstanbul 2010, 21-30.

J. G. C. Anderson, A Summer in Phrygia: I, JHS 17, 1897, 396-424.

K. Belke - N. Mersich, Phrygien und Pisidien (TIB 7), Wien 1990.

D. Berndt, Midasstadt in Phrygien. Eine sagenumwobene Stätte im Anatolischen Hochland, Mainz 2002.

S. Berndt, The historical geography of Phrygia. The Settlement of Leonton Kephalai, Orbis Terrarum 16, 2018, 23-53.

T. Christian, Gebildete Steine. Zur Rezeption literarischer Techniken in den Versinschriften seit dem Hellenismus (Hypomnemata 197), Göttingen 2015.

A. Coşkun, Dionysiac Associations among the Dedicants of Hosios kai Dikaios. Revisiting Recently Published Inscriptions from the Mihalıççık District in North-West Galatia, Gephyra 19, 2020, 111-133.

Th. Drew-Bear, Nouvelle Inscriptions de Phrygie, Zutphen 1978. Empedocles, The Extant Fragments, M. R. Wright (Hrsg.), New Haven-London 1981.

E. Erten, Dorylaion'dan Yeni Bir Hosios kai Dikaios Adağı ve Atlı Tanrı Üzerine Bazı Düşünceler, Phaselis IV, 2018, 199-217.

Etymologicum Magnum, T. Gaisford (Hrsg.), Oxford 1848.

P. Frei, Phrygische Toponyme, EA 11, 1988, 9-34.

V. Garulli, Byblos lainee. Epigrafia, letteratura, epitafio (Eikasmos, Studi 20), Bologna 2012.

F. T. Gignac, A grammar of the Greek Papyri of the Roman and Byzantine Periods I. Phonology, Milan 1976.

H. Güney, New Inscriptions from Northeast Phrygia: The Cult of Hosios and Dikaios, Gephyra 15, 2018, 101-117. 
Haspels 1971

Haspels 2009

Hemberg 1959

Herrmann 1962

I.Pessinous

İznik 2006

Keil - Premerstein 1908

Körte 1898

Kubińska 1968

Le Bas - Waddington

1972

LfgrE

LGPN VC

Lochman 2003

Magie 1950

MAMA I

MAMA V

MAMA VI
C. H. E. Haspels, The Highlands of Phrygia: Sites and Monuments, 2 cilt, Princeton/New Jersey 1971.

C. H. E. Haspels, Frigya. Midas Kenti Kazıları ve Dağlık Frigya Bölgesi'ndeki Araştırmalar (Hrsg.: D. Berndt, mit der Hilfe von H. Çambel), İstanbul 2009.

B. Hemberg, ANA $\Xi$, ANA $\Sigma \Sigma$ A und ANAKE $\Sigma$ als Götternamen unter besonderer Berücksichtigung der attischen Kulte, Uppsala 1959.

P. Herrmann, Ergebnisse Einer Reise in Nordostlydien (Denkschriften der Österreichische Akademie der Wissenschaften. Philosophisch-historische Klasse 80), Wien 1962.

J. Strubbe, The Inscriptions of Pessinous (IK 66), Bonn 2005.

E. İznik, Roma İmparatorluğu Döneminde Frigya Epiktetos Merkezlerinde (Dorylaeum, Nacolea, Midaeum, Cotiaeum, Aezani) Sosyopolitik, Sosyoekonomik ve Sosyokültürel Yapı (Unpublizierte Dissertation), Eskişehir 2006.

J. Keil - A. von Premerstein, Bericht über eine Reise in Lydien und der südlichen Aiolis, Wien 1908.

A. Körte, Kleinasiatische Studien III. Die phrygischen Felsdenkmäler, MDAI 23, 1898, 80-153.

J. Kubińska, Les monuments funéraires dans les inscriptions grecques de l'Asie Mineure, Warsaw 1968.

P. Le Bas - W. H. Waddington, Inscriptions grecques et latines recueillies en Gréce et en Asie Mineure, Hildesheim-New York 1972 (Nachdruck Paris 1870).

B. Snell (Begründer), Lexikon des frühgriechischen Epos, Bd.

4. P- $\Omega$ [rhô-oméga], Göttingen 2010.

J. -S. Balzat - R. W. V. Catling - É. Chiricat - Th. Corsten, A Lexicon of Greek Personal Names, Vol. VC: Inland Asia Minor, Oxford 2018.

Th. Lochman, Studien zu kaiserzeitlichen Grab- und Votivereliefs aus Phrygien, Basel 2003.

D. Magie, Roman Rule in Asia Minor. To the End of the Third Century After Christ I, Princeton 1950.

W. M. Calder, Monumenta Asiae Minoris Antiqua I, Manchester 1928.

C. W. M. Cox - A. Cameron, Monuments from Dorylaeum and Nacolea (MAMA V), Manchester 1937.

W. H. Buckler - W. M. Calder, Monuments and Documents from Phrygia and Caria (MAMA VI), Manchester 1939. 
MAMA X

Mitchell 1993

Mitchell - French 2019

Mommsen 1897

Naour 1981

Onur - Kılıç-Aslan 2021

Perrot 1872

Pfuhl - Möbius 1979

Pircher 1979

Polat 2009

Ramsay 1887

Ramsay 1897

Ramsay 1908

Ramsay 1918

Ricl 1990

Ricl 1991

Ricl 1992a

Ricl 1992b

Ricl 2008
B. Levick - S. Mitchell et alii (eds.), Monuments from Appia and the Upper Tembris Valley, Cotiaeum, Cadi, Synaus, Ancyra Sidera and Tiberiopolis (MAMA X), London 1993.

S. Mitchell, Anatolia: Land, Men, and Gods in Asia Minor Volume I: The Celts in Anatolia and the Impact of Roman Rule, Oxford 1993.

S. Mitchell - D. French, The Greek and Latin Inscriptions of Ankara (Ancyra), Vol. II: Late Roman, Byzantine and other texts, München 2019.

Th. Mommsen, Epinikos, Hermes 32, 1897, 660-663.

Ch. Naour, Inscriptions du Moyen Hermos, ZPE 44, 1981, 11-44.

F. Onur - S. Kılıç-Aslan, New Inscriptions from Aloanda in Lycia, Gephyra 21, 2021, 1-45.

G. Perrot, Exploration archéologique de la Galatie et de la Bithynie, d'une partie de la Mysie, de la Phrygie, de la Cappadoce et du Pont, Paris 1872.

E. Pfuhl - H. Möbius, Die ostgriechischen Grabreliefs II, Mainz 1979.

J. Pircher, Das Lob der Frau im vorchristlichen Grabepigramm der Griechen, Innsbruck 1979.

Y. Polat, Dağlık Phrygia Bölgesi Yerleşim Tiplerinin Coğrafi Bilgi Sistemleri ile İncelenmesi, in: A. Çabuk - F. Alanyalı (Hrsgg.), Kültür Varlıklarının Belgelenmesi, Eskişehir 2009, 104-118.

W. M. Ramsay, The Cities and Bishoprics of Phrygia (Continued), JHS 8, 1887, 461-519.

W. M. Ramsay, The Cities and Bishoprics of Phrygia. Vol. I, Part 2. The Lycos Valley and South-western Phrygia, Oxford 1897.

W. M. Ramsay, Luke the Physician, and Other Studies in the History of Religion, London 1908.

W. M. Ramsay, The Utilisation of Old Epigraphic Copies, JHS 38, 1918, 124-192.

M. Ricl, Le sanctuaire des dieux Saint et Juste à Yaylababa Köyü, Ziva Ant 40, 1990, 158-177.

M. Ricl, Hosios Kai Dikaios. Premiere partie: Catalogue des inscriptions, EA 18, 1991, 1-70.

M. Ricl, Hosios Kai Dikaios. Seconde partie: Analyse, EA 19, 1992, 71-103.

M. Ricl, Hosios Kai Dikaios. Nouveaux Monuments, EA 20, 1992, 95-100.

M. Ricl, Newly published and unpublished Inscriptions for Hosios and Dikaios and their Contribution to the Study of the Cult, in: E. 
Robert 1937

Robert 1980

Schwyzer 1939

Schuler 1998

SGO I

SGO II

SGO III

SGO IV

Staab 2018

Steuart 1842

TAM V,2

Tamsü Polat 2018

Texier 1839

Tucker 1990

Tüfekçi Sivas 1999
Winter (Hrsg.), Vom Euphrat bis zum Bosporus. Kleinasien in der Antike. Festschrift für Elmar Schwertheim zum 65. Geburtstag (Asia Minor Studien 65), Bonn 2008, 563-579.

L. Robert, Études anatoliennes. Recherches sur les inscriptions grecques de l'Asie Mineure, Paris 1937.

L. Robert, À travers l'Asie Mineure: poètes et prosateurs, monnaies grecques, voyageurs et géographie, Paris 1980.

E. Schwyzer, Griechische Grammatik I. Allgemeiner Teil, Lautlehre, Wortbildung, Flexion, München 1939.

Chr. Schuler, Ländliche Siedlungen und Gemeinden im hellenistischen und römischen Kleinasien, München 1998.

R. Merkelbach - J. Stauber, Steinepigramme aus dem griechischen Osten I: Die Westküste Kleinasiens von Knidos bis Ilion, München-Leipzig 1998.

R. Merkelbach - J. Stauber, Steinepigramme aus dem griechischen Osten II: Die Nordküste Kleinasiens (Marmarameer und Pontos), München-Leipzig 2001.

R. Merkelbach - J. Stauber, Steinepigramme aus dem griechischen Osten III: Der ferne Osten und das Landesinnere bis zum Tauros, München-Leipzig 2001.

R. Merkelbach - J. Stauber, Steinepigramme aus dem griechischen Osten IV: Die Südküste Kleinasiens, Syrien und Palaestina, München-Leipzig 2002.

G. Staab, Gebrochener Glanz. Klassische Tradition und Alltagswelt im Spiegel neuer und alter Grabepigramme des griechischen Ostens, Berlin-Boston 2018.

J. R. Steuart, A description of some ancient monuments, with inscriptions, still existing in Lydia and Phrygia, several of which are supposed to be tombs of early kings, London 1842.

P. Herrmann, Tituli Asiae Minoris V. Tituli Lydiae linguis Graeca et Latina conscripti. Fasc. II: Regio septentrionalis ad occidentem vergens, Wien 1989.

R. Tamsü Polat, Yazılıkaya/Midas Vadisi Akpara Kale Mezarları, Olba 26, 2018, 249-284.

C. Texier, Description de l'Asie Mineure: faite par ordre du gouvernement français en 1833-1837, Première partie, Paris 1839.

E. F. Tucker, The Creation of Morphological Regularity. Early Greek Verbs in -éō, -áó, -óō, -úō and -íō, Göttingen 1990. (https:// opacplus.bsb-muenchen.de/title/BV002473959) 20.04.2021.

T. Tüfekçi Sivas, Eskişehir-Afyonkarahisar-Kütahya il sınırları içindeki Phryg kaya anıtları (Anadolu Üniversitesi Yayınları No 1156), Eskişehir 1999. 
Tüfekçi Sivas 2007

Tüfekçi Sivas 2012

Tüfekçi Sivas - Sivas 2007

Waelkens 1986

Wallner 2011

Zgusta 1964

T. Tüfekçi Sivas, Batı Frigya'da Frig Yerleşmeleri ve Kaya Anıtlarının Araştırılması, in: H. Sivas - T. Tüfekçi Sivas (Hrsgg.), Friglerin Gizemli Uygarlığı/The Mysterious Civilization of the Phrygians, İstanbul 2007.

T. Tüfekçi Sivas, Frig Vadileri ve Kutsal Yazılıkaya-Midas Kenti, in: T. Tüfekçi Sivas - H. Sivas (Hrsgg.), Frigler/Phrygians. Midas'in Ülkesinde, Anttların Gölgesinde/In the Land of Midas, In the shadow of monuments, İstanbul 2012, 112-159.

T. Tüfekçi Sivas - H. Sivas, Frig Vadileri (Friglerden Türk Dönemine Uzanan Kültürel Miras), Eskişehir 2007.

M. Waelkens, Die kleinasiatischen Türsteine: Typologische und epigraphische Untersuchungen der kleinasiatische Grabreliefs mit Scheintür, Mainz am Rhein 1986.

Ch. Wallner, Die Inschriften des Museums in Yozgat, Wien 2011.

L. Zgusta, Kleinasiatische Personennamen, Prag 1964.

\section{Dağlık Phrygia'dan Yeni Yazttlar}

Özet

$\mathrm{Bu}$ makalede Anadolu Üniversitesi Arkeoloji Bölümü öğretim üyeleri Rahşan Tamsü-Polat ve Yusuf Polat tarafından gerçekleştirilen Frig vadisi yüzey araştırmalarında keşfedilen ve bir tanesi (no. 5) daha önceden yayımlanmış olan 6 adet Yunanca yazıt tanıtılmaktadır. Yazıtların ikisi (no. 1-2) Tanrı Megistos Hosios'a sunulan adak yazıtlarıdır. Bu tanrı ile büyük olasılıkla Dorylaion ve çevresinden çok iyi bilinen Hosios ve Dikaios isimli tanrılar kastediliyor olmalıdır. Fakat tanrı buradaki yazıtlarda sadece tek bir tanrı olarak karşımıza çıkmaktadır ve isminin "Dikaios" kısmı yoktur. Bu tanrının Megistos epithet'i ilk defa bu yazıtlar sayesinde ortaya çımaktadır. Yazıtların iki tanesi ise epigram'lardan (no. 3-4) oluşmaktadır. İlk epigram bir pentametron ve altı heksametron vezninde kaleme alınmış olup eser Pasikrates isimli birisi tarafından diktirilmiştir. $\mathrm{Bu}$ yazıtta Pasikrates'in Efendisi Letoides'e (Leto'nun oğlu), yani Apollon'a bir teşekkür şiiri sunduğunu görüyoruz. Şiirde Pasikrates eşi Dada'nın yanı sıra çok sayıdaki çocuklarının da isimlerini ve özelliklerini saymaktadır. Makalemizdeki diğer epigram ise bir mezar şiiri/ağıtı olup Karikos isimli birisine aittir. Şiire göre eserin üzerinde vaktiyle iki adet aslan heykeli de yer almaktaydı. Mezar soyguncularına karşı koruyucu bir tedbir olarak konulan aslanlar bugün kayıp olsa da stel üzerinde görülebilen dübel delikleri şiirde bahsedilen bu aslanların heykellerinin yerleștirilmesi için kullanılmış olsa gerektir. Şiirde bir kelime oyunu vardır ve ölen kişinin (bu) vahşi hayvanlarla (Yunancası $\theta \eta ́ \rho)$ aynı adı taşıyan bir işi ( $\theta \dot{\rho} \rho \alpha=$ avcılık) yaptığından söz edilmektedir. Karikos'un bir avcı olması muhtemeldir. Son iki yazıt (no. 5-6) düz mezar yazıtı olup ilki Solon ve Nana için çocukları Menodoros ve diğer bir oğlu tarafindan yaptırılmıştır; diğer yazıt ise fragmandır. Eserlerin hepsi Roma İmparatorluk Dönemi’nin 2. veya 3. yüzyıllarındandır. Yazıtların Türkçe çevirileri şöyledir:

1. Andr - - - oğlu, brabeutes - - -on, Tanrı Megistos Hosios için (bu) adağı dikti.

2. Tyrannos oğlu rahip Polykrates? Tanrı Megistos Hosios'a adağı (sundu).

3. Pasikrates (bu altarı adadı). 
Çünkü onu Efendi Letoides (= Leto'nun oğlu = Apollon) atalarından beri seviyordu ve onu tüm ölümlüler arasında açık ara en iyi kişi yapmıştı. Eşi Dada ile beraber asil çocuklar dünyaya getirdi: bilgelikte yaşından çok daha olgun olan Papylos'u, sonra Leonti(o)s'u, Tanrı (görevine) kutsanmış Pasikrates'i ve sevgiyle özlemi duyulan Patriki(o)s'u ve Gregori(o)s'u (ve) hepsinden daha sevimli bir şekilde öne çıkan Antiokhos'u, çok saygıdeğer kızları Domna'yı ve beraberinde Stratonike'yi.

4. Bu mezarın üzerinde iki aslan görüyorsun, dağlarda yaşayan hayvanları; isimleriyle aynı anlama gelen bir işi yapan kişiyi bekliyorlar. Annem Demetria'dan doğmayım, babam ise Diomedes idi. Adım Karikos'tu, bütün ölümlülere derman olan! Hayattayken ve aklım yerindeyken yaptırdı(m) bu parlak taştan mezar anıtını.

5. - - - s ve Menodoros babaları Solon için ve (anneleri) Nana için (hatıraları nedeniyle bu mezarı yaptirdilar.)

6. - - - - anisı nedeniyle - - -

Anahtar Sözcükler: Yazılıkaya, Eskişehir, Metropolis, Yunanca yazıtlar, adak yazıtları, Theos Megistos Hosios, brabeutes, epigram, Letoides.

\title{
New Inscriptions from the Highlands of Phrygia
}

\begin{abstract}
In this article, we present six inscriptions (one has already been published) discovered in the course of the on-going surveys in the Phrygian Highlands conducted by Rahşan Tamsü-Polat and Yusuf Polat from the archaeology department of Anadolu University (Eskişehir). Two of the inscriptions are ex-votos dedicated to Megistos Hosios, which are most likely to be associated with Hosios and Dikaios worshipped predominantly in Dorylaion and in its environs. In both of these dedications, the god appears as one deity and does not bear the name 'Dikaios', but has the epithet of Megistos, attested here for the first time. Two of the inscriptions are epigrams. The first is an epigram comprising of one pentamer and 6 hexameters in verse. The inscribed monument is erected by a certain Pasikrates who expresses his gratitude through this poem to his Lord Letoides (Leto's son) i.e. to Apollon. In this epigram, Pasikrates list the names of his wife and many children along with their characteristics. The other poem is of the funerary epigram genre, belonging to a man called Karikos. According to the epigram, two lions were standing on the top of the funerary monument, apparently serving an apotropaic function. Even though these lions are missing today, the dowel holes visible on the monument were almost certainly employed to secure the recorded statues of two lions. In the epigram there is a sort of wordplay and the deceased seemingly performed something (i.e. $\theta \eta \dot{\rho} \alpha=$ hunting) which bears the same name with these wild animals ( $\theta$ п́ $\rho)$, and this possibly indicates that Karikos was a hunter. The last two inscriptions (nos 5-6) are funerary inscriptions. The first is inscribed for Solon and Nana by their child Menodoros. The other is fragmentary. All of these inscriptions date from the Roman Imperial Period $\left(2^{\text {nd }}\right.$ and $3^{\text {rd }}$ century A.D.).
\end{abstract}

Keywords: Yazılıkaya, Eskişehir, Metropolis, Greek Inscriptions, votive inscriptions, Theos Megistos Hosios, brabeutes, epigrams, Letoides. 